\title{
THE EFFECT OF CARDIAC REHABILITATION ON 30-DAY HOSPITAL READMISSION RATES
}

A dissertation submitted to the Kent State University College and School of Education, Health, and Human Services in partial fulfillment of the requirements for the degree of Doctor of Philosophy

Allan Shook

May 2015 
(C) Copyright, 2015 by Allan Shook All Rights Reserved 
A dissertation written by

Allan Shook

B.S., Kent State University, 2000

M.A., Kent State University, 2006

Ph.D., Kent State University, 2015

Approved by

, Director, Doctoral Dissertation Committee

Ellen Glickman

, Member, Doctoral Dissertation Committee

Jacob Barkley

, Outside Member, Doctoral Dissertation

John Gunstad Committee

Accepted by

, Director, School of Health Sciences

Lynne E. Rowan

, Dean, College of Education, Health and

Daniel F. Mahony Human Services 
SHOOK, ALLAN, Ph.D., May 2015

HEALTH SCIENCES

\section{THE EFFECTS OF CARDIAC REHABILITATION ON 30-DAY HOSPITAL READMISSION RATES (90 pp.)}

Director of Dissertation: Ellen Glickman, Ph.D.

Readmission rates at 30 days are considered a hospital quality indicator, and previous research suggests that up to $76 \%$ of 30 -day readmissions could be prevented. The purpose of this study was to compare 30-, 60-, and 180-day readmission and short-term observation (STO) rates for participants and non-participants in Phase II cardiac rehabilitation (CR), and to determine predictors for readmission, STO and CR participation. Ninety subjects (participants $n=45$; non-participants $n=45$ ) retrospectively participated in the study. All subjects were referred to CR following a qualifying diagnosis. An additional 131 subjects were retrospectively studied to quantify predictors for readmission, STO, and participation in CR. Thirty-day readmission rates approached statistical significance and were higher for non-participants than participants $(p=.064)$. Sixty-day readmission rates were higher for non-participants but not statistically significant. One hundred eighty-day readmission rates were significantly higher for non-participants than participants $(p=.014)$. Thirty- and 60-day STO rates were slightly higher for non-participants but not statistically significant. One hundred eighty-day STO rates were statistically higher for participants than non-participants $(\mathrm{p}=$ .027). Predictors for readmission and STO were varied, based on timeframe. Being smoke-free, non-hypertensive, married, and not having a myocardial infarction (MI) at admission were significant predictors for enrolling in CR. CR appears to decrease the 
likelihood for readmission at 180 days. STO rates were higher at 180 days for CR participants, perhaps indicating a need for increased monitoring without rehospitalization. Predictors for readmission and STO were widely varied, necessitating further research. 


\section{ACKNOWLEDGMENTS}

This project would not have been possible without the encouragement and support of many people. I would like to thank my academic advisor and director of my dissertation committee, Dr. Ellen Glickman. Without your guidance, mentoring, and encouragement, I would not have been able to complete this project. I thank you for all you have done for me academically and professionally. I would also like to thank the other committee members: Dr. Jacob Barkley for his assistance with data analysis, interpretation, and recommendations on how to best "tell the story;" Dr. John Gunstad for his insight and critical analysis of the study's findings; and Dr. Dianne Kerr for her unique perspectives on what the findings mean in the bigger picture.

Sharon Smith did a fantastic job of editing and formatting this paper to meet APA and KSU guidelines for submission. I appreciate her patience with my many gaffes in formatting and the outstanding attention to detail during the editing process.

The wonderful staff of Cardiopulmonary Rehab and the Heart Failure Clinic, along with the administration of the Heart and Vascular Center, offered tremendous support throughout this project. Many times they covered shifts while I was collecting or analyzing data, and they offered kind words and encouragement throughout the process.

Finally, I would like to thank my family: my Mom and Dad; my wife, Tracy; and my daughters, Madison and Emily. They were my rocks throughout this process and they backed me every step of the way. They inspired me to continue and persevere through this process, and cheered me on every step of the way. They sacrificed so much over the past year-and-a-half so that I could complete this project and did so very selflessly, 
without complaint. Tracy was, and is, my pillar of strength when I doubted myself or got discouraged during the process. She refused to let me give up at any step of the way, and always reminded me of "the light at the end of the tunnel." Words cannot begin to describe how much your love and support through this project means to me. To my Mom and Dad, I hope that you are proud of me as you look down on me from Heaven.

To everyone involved, please know that on May 8, 2015, I will be taking you with me in my mind and heart as I graduate. Thank you to everyone who was involved, directly or indirectly, in this project. 


\section{TABLE OF CONTENTS}

ACKNOWLEDGMENTS iv

LIST OF TABLES viii

\section{CHAPTER}

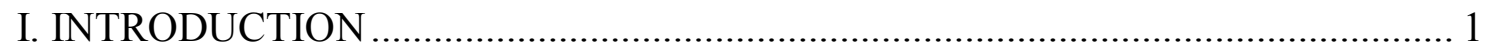

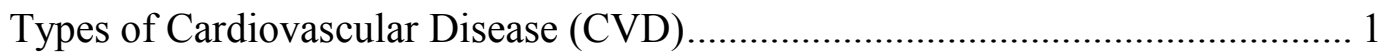

The Widespread Epidemic of CVD in the United States........................................ 1

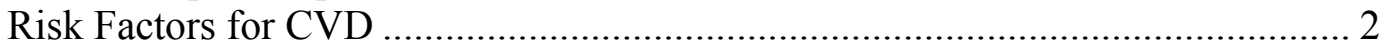

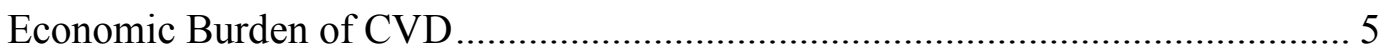

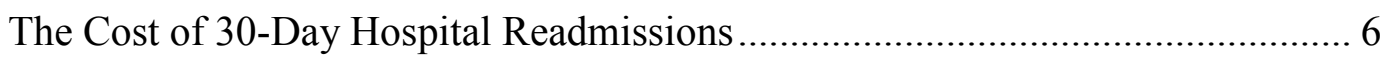

The Role of CR in Primary and Secondary Prevention of CVD ........................... 7

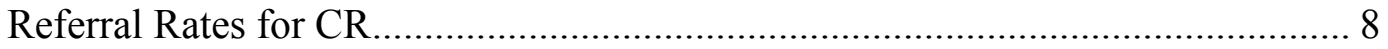

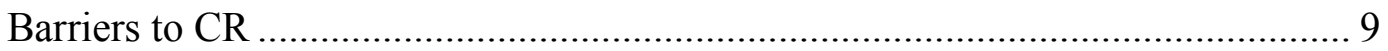

Increasing CR Referrals, Enrollment, and Participation....................................... 10

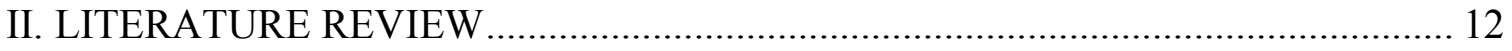

The Issue of 30-Day Hospital Readmissions .................................................... 12

Types of Hospital Readmissions ...............................................................12

Consequences of 30-Day Hospital Readmissions ......................................13

Incidence of 30-Day Hospital Readmissions for AMI ...................................15

Incidence of 30-Day Hospital Readmissions for PCI....................................16

Incidence of 30-Day Hospital Readmissions for CABG ……………….........17

Incidence of 30-Day Hospital Readmissions for STEMI ..............................18

Predicting 30-Day Hospital Readmissions .................................................19

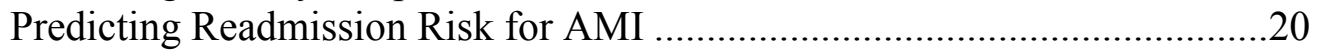

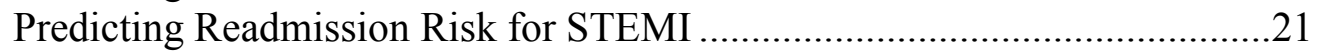

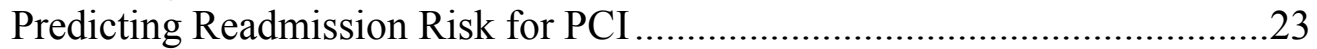

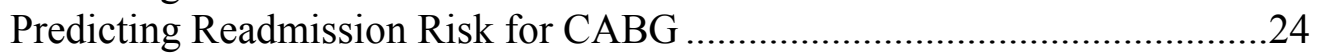

Private Insurance Versus Public Insurance.................................................24

The Relationship Between LOS and 30-Day Readmission Rates ..................27

30-Day Episodes of Care as a Quality Metric …………….........................29

Preventing 30-Day Hospital Readmissions ........................................................ 30

Cardiac Rehabilitation Reducing Hospitalizations .........................................34

Cardiac Rehabilitation Beginning at the Bedside..............................................35

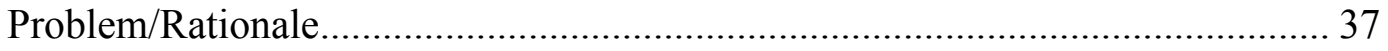

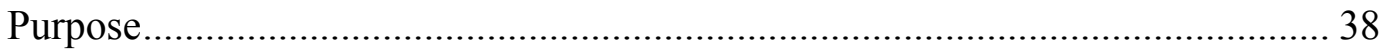




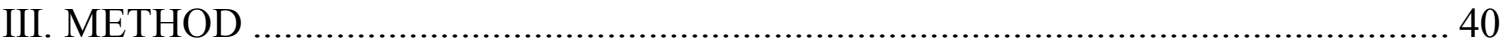

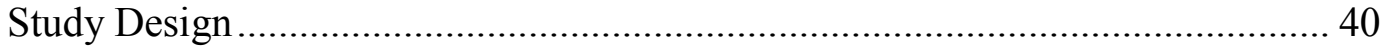

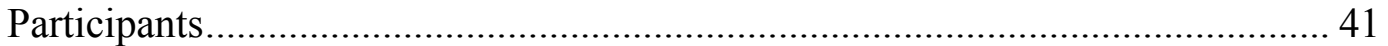

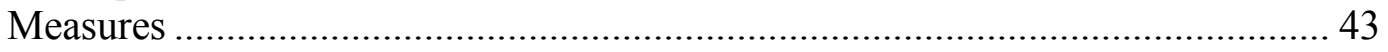

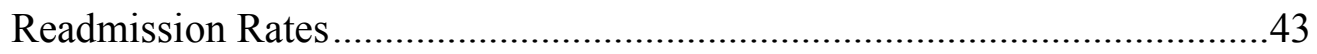

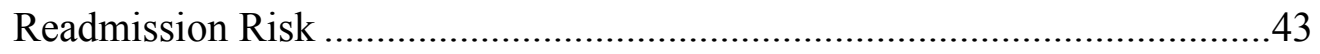

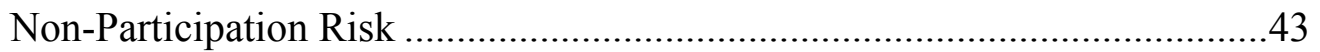

Demographic and Medical History............................................................

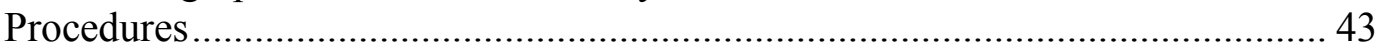

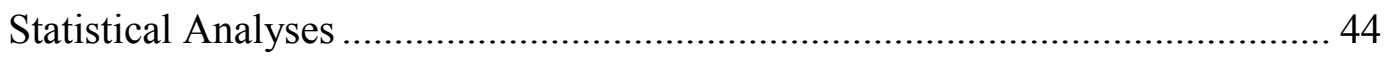

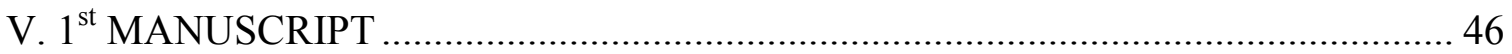

The Effect of Cardiac Rehabilitation on 30-, 60-, and 180-Day Unplanned Hospital Readmission and Short-Term Observation Rates .................................. 46

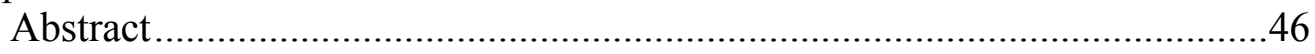

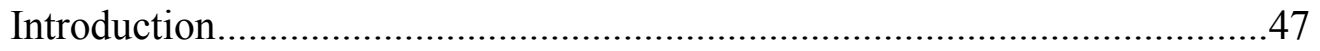

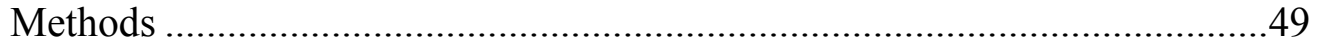

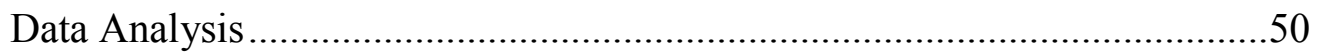

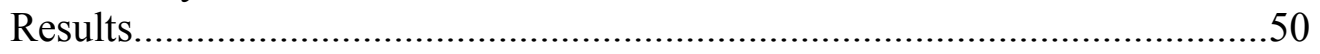

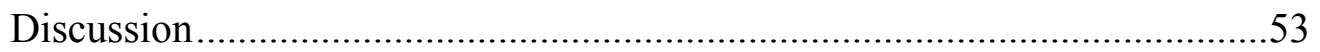

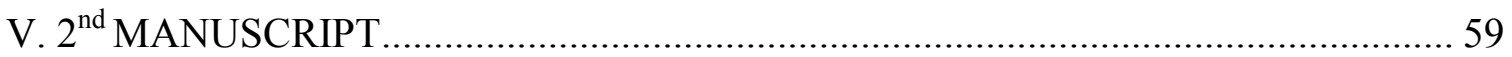

Predictors for Participation, Readmission and Short-Term Observation at 30, 60, and 180 Days Post-Hospitalization in Cardiac Rehabilitation Participants

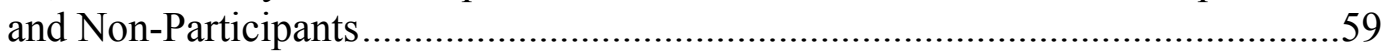

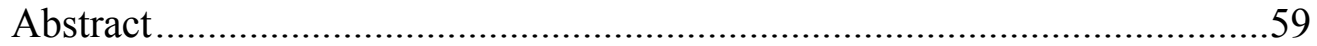

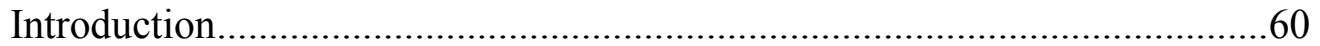

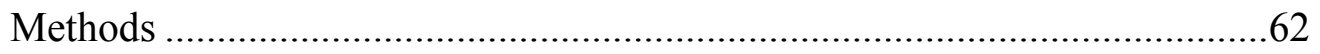

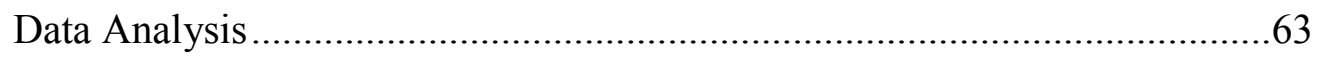

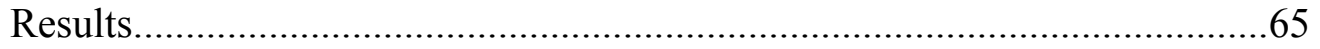

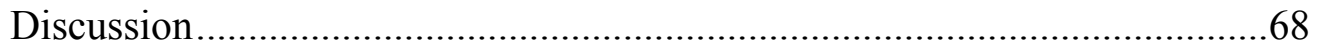

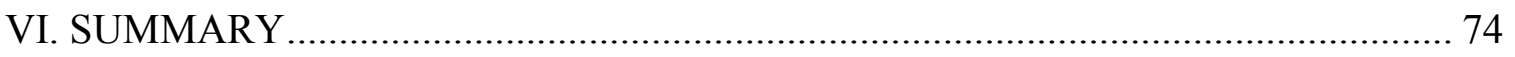

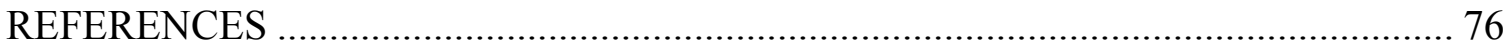




\section{LIST OF TABLES}

Table $\quad$ Page

1. Demographic and Readmission Data For Participants and Non-Participants ...... 42

2. Relative Risk Estimate By Participation Status and Timeframe ....................... 53

3. Demographic Data for Entire Cohort of Participants and Non-Participants......... 64

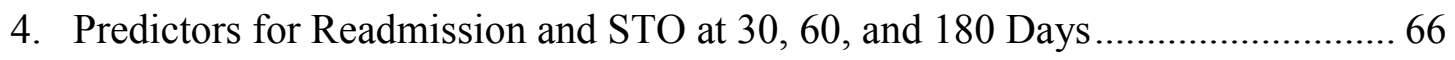

5. Predictors for Participation in Cardiac Rehabilitation .................................... 68 


\section{CHAPTER I}

\section{INTRODUCTION}

\section{Types of Cardiovascular Disease (CVD)}

Cardiovascular disease (CVD) is an encompassing term that refers to many conditions of the heart and blood vessels (American Heart Association, 2013; McGill et al., 2000; Sniderman, Pederson, \& Kjekshus, 1997; Vogel, 1997; Zhu et al., 2001). Coronary artery disease (CAD) is a condition affecting the coronary arteries that supply the heart with blood and oxygen. The primary cause of CAD is the progressive aggregation of plaque in the coronary arteries, a process called atherosclerosis (American Heart Association, 2013). Atherosclerosis causes a narrowing in the lumen of the arteries, leading to reduced blood flow. As blood flow is reduced, pressure within the coronary arteries can increase, causing plaque, which has built up in the arteries to become unstable, and possibly rupture. When plaque ruptures, the body attempts to repair the damage from the rupture, so a clot develops in the area, causing a myocardial infarction (MI).

\section{The Widespread Epidemic of CVD in the United States}

CVD remains an epidemic that affects the United States, with evidence showing the problem is only getting worse. According to data from the American Heart Association (AHA, 2012), one-third of all deaths in the United States were caused by CVD, and one-sixth of all deaths were caused by CAD. Approximately 2,200 Americans die from CVD each day, equal to one death every 39 seconds (AHA, 2012). African American males continue to have the highest rate of death from CVD, whereas Caucasian 
females have the lowest rate of death from CVD (Centers for Disease Control [CDC], 2010). Additionally, heart failure (HF) was listed as a secondary or contributing factor to death in over 281,000 deaths in 2012, and by 2030, more than 8 million Americans will be diagnosed with HF (CDC, 2010).

Mortality rates have declined by 30\% from 1998-2008 (AHA, 2012). Evidence based medical therapies such as beta-blockers, angiotensin converting enzyme (ACE) inhibitors, and statins, as well as secondary risk factor reduction are the primary reasons for this (Ford et al., 2007). Despite this, the AHA (2012) predicts there will be 785,000 new cardiac events, and 470,000 repeat cardiac events. An additional 195,000 Americans will suffer a silent (i.e., symptom-free) myocardial infarction (MI) this year.

\section{Risk Factors for CVD}

Risk factors for CVD are typically classified as modifiable or non-modifiable. Non-modifiable risk factors include age, gender, and family history (American College of Sports Medicine [ACSM], 2010). According to the ACSM (2010), modifiable risk factors include hypertension, tobacco abuse, diabetes, obesity, metabolic syndrome, sedentary lifestyle, stress, and depression. Other emerging risk factors include C-reactive proteins (CRP), homocysteine, and lipoprotein a (Lp[a]) levels (ACSM, 2010).

One-third of American adults over age 20 are hypertensive, with similar rates between men and women; African Americans have the highest rate of hypertension, at $44 \%$ (Howard et al., 2006). Although $80 \%$ of those with hypertension are aware of their condition, only $71 \%$ are on medication and less than half of those who know they are 
hypertensive have adequate blood pressure (BP) control (Ostchega, Yoon, Hughes, and Louis, 2008).

According to the CDC (2010), smoking rates have declined over the past four decades. Eaton et al. (2010) reported $21 \%$ of men and $17.5 \%$ of women over the age of 18 smoke; $19.5 \%$ of 9-12 graders smoke (CDC, 2010). Exposure to second-hand smoke in the non-smoking population has decreased from $52.5 \%$ to $40.1 \%$ (CDC, 2010; Schiller, Lucas, Ward, \& Peregoy, 2012). This may be a result of changes in societal attitudes toward cigarette smoking, improved education, and the banning of smoking in public places in 25 states and the District of Columbia (CDC, 2010).

More than 33 million Americans, roughly 15\% of the total population, have a serum total cholesterol greater than $240 \mathrm{mg} / \mathrm{dl}$ (Kuklina, Shaw, \& Hong, 2011). Other components of cholesterol: high-density lipoprotein (HDL), low-density lipoprotein (LDL) and triglyceride levels, can affect cardiovascular risk as well.

The incidence of diabetes continues to grow in the United States. Over 18 million Americans are diabetic, with a majority having Type II (non-insulin dependent) diabetes (CDC, 2010). There are over 7 million Americans with undiagnosed diabetes and 36.8\% of the population has abnormal fasting glucose levels (National Health and Nutrition Examination Survey; National Heart Lung and Blood Institute; National Center for Health Statistics, 2005-2008). Diabetes is more common in minorities than in Caucasians (Cowie et al., 2006), and the prevalence of diabetes has increased in conjunction with increases in obesity levels (Ford, 2011; Ogden, Carroll, Kit, \& Flegal, 2012). 
Nearly 150 million American adults over age 20 (67.3\%) are either overweight or obese. Thirty four percent of all adults are obese, regardless of ethnicity (Flegal, Carroll, Ogden, \& Curtin, 2010). Nearly one-third of children aged 2-19 are overweight, and 17\% of children aged 2-19 are obese, with Mexican Americans and African American girls more affected than other ethnicities (Flegal et al., 2010). The past three decades have seen obesity rates in children aged 6-11 go from 4\% to 20\% (Ogden et al., 2012). According to the National Health and Nutrition Examination Survey (Ford, 2011), average caloric consumption has increased by $22 \%$ in women, and $10 \%$ in women from 1971-2004 without an equivalent increase in calories burned through physical activity.

Metabolic syndrome is a pre-cursor to Type II diabetes. It is classified by elevated $\mathrm{BP}$ ( $>130 \mathrm{mmHg}$ systolic or $>85 \mathrm{mmHg}$ diastolic), low $\mathrm{HDL}$ ( $<40 \mathrm{mg} / \mathrm{dl}$ for males; $<50 \mathrm{mg} / \mathrm{dl}$ for females); elevated triglycerides ( $>150 \mathrm{mg} / \mathrm{dl})$, high-risk waist circumference ( $>40$ inches for males, $>35$ inches for females) or elevated fasting glucose (>100 mg/dl). To meet the criteria for Metabolic Syndrome, a person must meet at least three of the five criteria (ACSM, 2010). Thirty four percent of Americans meet the criteria for Metabolic Syndrome, with slightly more men than women meeting criteria (Ervin, 2009).

One-third of all Americans are considered sedentary (Schiller et al., 2012), which is the failure to engage in planned physical activity a minimum of three days per week for a period of three months (ACSM, 2010). ACSM recommends planned physical activity on most, if not all days of the week. Despite these recommendations, Eaton et al. (2010) 
reported that $30 \%$ of $9-12$ grade girls and $20 \%$ of boys stated they had not engaged in any physical activity within the past seven days.

Stress and depression are both now recognized as extremely powerful risk factors for CVD (Doe, 2009; Rozanski, Blumenthal, Davidson, Saab, \& Kubzansky, 2005; Rozanski, Blumenthal, \& Kaplan, 1999). Both stress and depression are highly individualized and can be difficult to quantify. Nonetheless, recent research has shed light on the potential impacts each has on CVD. In addition, several studies (Carney, Freedland, Miller, \& Jaffe, 2002; Carney, Freedland, Sheline, \& Weiss, 1997; Lett et al., 2004; Rugulies, 2002; Wulsin \& Singal, 2003) have demonstrated the prognostic value of depression in relation to all-cause mortality in coronary heart disease (CHD).

\section{Economic Burden of CVD}

The cost of CVD is staggering. In 2008, CVD accounted for $\$ 179$ billion in direct costs and another $\$ 118.5$ billion through indirect costs such as lost work, and productivity (Agency for Healthcare Research and Quality, Medical Expenditure Panel Survey, 2008). CVD required more healthcare dollars than any other disease, including cancer, and CVD accounted for 16\% of all healthcare dollars spent in 2008 (Agency for Healthcare Research and Quality, Medical Expenditure Panel Survey, 2013; American Cancer Society, 2015). It is estimated that between $\$ 201$ billion and $\$ 228$ billion were spent on cancer in 2008 (American Cancer Society, 2015; Yabroff, Lund, Kepka, \& Mariotto, 2011). It is estimated that between 2012-2030, the direct cost of CVD will nearly triple, from $\$ 309$ billion to $\$ 834$ billion, and that the indirect cost of CVD will increase by more than $50 \%$, from $\$ 185$ billion to $\$ 284$ billion (Heidenreich et al., 2011). 
The end result is that by 2030 , over $\$ 1$ trillion will be spent on CVD. In light of the escalating costs of healthcare, both private insurance companies and the Centers for Medicare and Medicaid Services (CMS) are exploring ways to contain costs.

\section{The Cost of 30-Day Hospital Readmissions}

One of the measures being examined as part of the Patient Protection and Affordable Care Act (PPACA) is reducing the incidence of 30-day hospital readmissions (PPACA, 2010). In a report released by the Robert Woods Johnson Foundation (Goodman, Fisher, Chang, Raymond, \& Bronner, 2013), approximately 1 in 5 Medicare discharges results in a 30-day readmission, costing $\$ 26$ billion. It is estimated that nearly $\$ 17$ billion worth of hospital readmissions could be prevented (Robert Woods Johnson Foundation, 2013). Even still, there is debate as to what the cost savings could be with reducing 30-day readmissions, particularly because it is difficult at times to ascertain which readmissions are truly preventable and because 30 -day readmission rates vary greatly throughout the country. Summit County in Ohio reports a 30-day readmission rate of $15.6 \%$ and now 30-day readmission rates are calculated by CMS. As of fiscal year 2013 (FY 2013), three diagnoses are being evaluated by CMS for excessive readmission rates: HF, pneumonia (PN), and acute myocardial infarction (AMI). Given that HF and AMI ultimately are part of a chronic disease (CVD), there is increasing emphasis on secondary disease prevention. One option for secondary prevention is cardiac rehabilitation $(\mathrm{CR})$. 


\section{The Role of CR in Primary and Secondary Prevention of CVD}

Primary prevention involves reducing the risk for initial presentation of disease, whereas secondary prevention focuses on reducing the risk for future occurrences or episodes of a chronic disease. There are many benefits of participating in a cardiac rehabilitation program.

Artham, Lavie, Milani, Chi, and Goldman (2008) noted a 35\% increase in metabolic equivalent (MET) levels, a 15\% increase in peak oxygen consumption $\left(\mathrm{VO}_{2 \text { peak }}\right)$, and an $11 \%$ increase in anaerobic threshold. Milani, Lavie, and Mehra (2004) found that individuals participating in cardiac rehabilitation see improvements in lipid profiles, including improved HDL levels, decreased LDL, triglyceride, and total cholesterol levels. CR was found to reduce body mass index (BMI) by $1.5 \%$, body fat by $5 \%$, and to decrease the incidence of Metabolic Syndrome by 37\% (Lavie, Morshedi-Meibodi, \& Milani, 2008; Milani \& Lavie, 2003).

CR also reduces inflammatory markers (Milani et al., 2004; Ali et al., 1998). High-sensitivity CRP (hs-CRP) was reduced by $40 \%$, along with a reduction in homocysteine levels and blood viscosity. Additionally, endothelial function improved with participation in CR (Hambrecht et al., 2000).

Numerous studies outline the psychological benefits of CR. Prior studies (Doe, 2009; Lucini et al., 2002) found that patients who participate in CR are significantly less depressed and have improved autonomic tone. Other studies (Artham et al., 2008;

Dimsdale, 2008; Lavie, Milani, Cassidy, \& Gilliland, 1999; Lichtman et al., 2008; Milani \& Lavie, 2007; Milani, Lavie, \& Cassidy, 1996; Rozanski et al., 2005) have reported 
reduced mortality rates as high as $70 \%$ and significantly reduced levels of anxiety, depression, hostility, somatization, and total stress, the reductions ranging from $40 \_70 \%$ in each area.

Participation in a CR program has shown significant effects on mortality and morbidity (Artham et al., 2008; Lavie et al., 2008; Squires, Gau, Miller, Allison, \& Lavie, 1990; Thomas et al., 2007; M. Williams et al., 2006). Lavie, Thomas, Squires, Allison, and Milani (2009) found a $20 \%$ reduction in total mortality and a $22 \%$ reduction in cardiac mortality. Additionally, there was a $25 \%$ reduction in fatal MI and a significant reduction in the incidence of sudden cardiac death (SCD) one year after participating in CR. Clearly, there are numerous benefits from participation in a CR program following a coronary event.

\section{Referral Rates for CR}

Despite the many benefits of $\mathrm{CR}$, it is a vastly underutilized service. According to the AHA Get With the Guidelines (AHA GWTG 2009; Brown et al., 2009), only 56\% of all eligible patients were referred to CR. Just over half (53\%) of patients with AMI were referred, $58 \%$ of patients with percutaneous coronary intervention (PCI) were referred, and $74 \%$ of patients with coronary artery bypass graft (CABG) surgery were referred. Even worse is the attendance rate in CR programs. There is an $18.7 \%$ attendance rate in CR programs in the United States (Lavie et al., 2009). Worldwide, only $15-30 \%$ of eligible patients attend CR (Neubeck et al., 2011). 


\section{Barriers to CR}

As with any exercise program, CR requires voluntarily participation. It requires commitment, time, energy, and must be a priority for an individual to enroll in it. There are many barriers in place preventing referral to, and enrollment in, CR. Barriers can be divided into three categories: patient-oriented factors, medical factors, and healthcare system factors (Ades, Waldmann, McCann, \& Weaver, 1992; AHA, 2011; Curnier, Savage, \& Ades, 2005; Grace et al., 2008; Hamilton \& Seidman, 1993; Mazzini, Stevens, Whalen, Ozonoff, \& Balady, 2008; Suaya et al., 2007; Valencia, Savage, \& Ades, 2011; Witt, Thomas, \& Roger, 2005). Patient-oriented factors include: female sex, advanced age, racial/ethnic minority group, lack of or limited health insurance, low socioeconomic status, low education level, low self-efficacy, low health literacy, perceived lack of need for $\mathrm{CR}$, language barriers, cultural beliefs that preclude participation in $\mathrm{CR}$, job-related and economic factors, limited social support, and domestic responsibilities (AHA, 2011). Patients with multiple, severe co-morbidities often are either not referred to CR, or choose not to attend because of their medical condition. Healthcare system factors can influence referral and enrollment into CR. These include: no CR referral, inefficient referral or enrollment process, lack of physician support for $\mathrm{CR}, \mathrm{CR}$ is not an offered service, not covered by insurance, lack of availability (i.e., classes not offered at convenient times), driving distance to and from CR, parking, and lack of ethnic or gender diversity (AHA, 2011). 


\section{Increasing CR Referrals, Enrollment, and Participation}

Higgins et al. (2008) discussed strategies for improving referral and enrollment into CR programs, including: automatic CR referrals upon hospital discharge, arranging for a representative from $\mathrm{CR}$ to facilitate enrollment, providing patients with options on $\mathrm{CR}$ programs in the area, giving written information and invitations to $\mathrm{CR}$, initiating enrollment into CR prior to hospital discharge (including appointment date for $\mathrm{CR}$ ), providing parking or transportation, and following up by phone or letter with patients who have not yet enrolled in CR.

Conversely, Dunlay et al. (2009) identified patient characteristics that increase the likelihood of CR referral and enrollment. Patients under the age of 65 were more likely to attend, and males were nearly twice as likely to attend CR as females. In addition, non-diabetics were 2.5 times more likely to participate in CR, and patients who had an ST-elevation myocardial infarction (STEMI) were 2.6 times more likely to enroll. Patients whose cardiologist practiced at the hospital to which they were admitted were 18 times more likely to enroll in CR, and if the patient received reperfusion therapy, they were 7 times more likely to participate in CR. Patients who had a prior MI were 4 times less likely to participate in $\mathrm{CR}$, and if they previously participated in CR, they were nearly 4 times less likely to enroll again. Inpatient $\mathrm{CR}$ referrals made patients 12 times more likely to attend. Psychosocial factors which made CR referrals and attendance more likely included feeling CR was a necessary component of recovery (12 times more likely), high perception of health prior to MI ( 7 times more likely), the ability to drive (6 
times more likely), completion of high school (3 times more likely), and highly valuing CR (2 times more likely). 


\section{CHAPTER II}

\section{LITERATURE REVIEW}

\section{The Issue of 30-Day Hospital Readmissions}

Healthcare is a $\$ 2.6$ trillion industry (CMS, 2012), with costs continuing to increase yearly. Over 31\% of all healthcare dollars ( $\$ 814$ billion) were spent on hospitals; it is by far the largest expense for insurance providers. An additional $\$ 515.5$ billion, nearly $20 \%$ of all healthcare expenditures, was spent on physician and/or clinical services (CMS, 2012). CMS reported other significant healthcare costs as administration and investments ( $\$ 407.6$ billion, $15.7 \%$ of healthcare dollars spent), medications $(\$ 259.1$ billion, $10 \%$ of healthcare dollars spent), professional and/or personal services ( $\$ 196.9$ billion, $7.6 \%$ of healthcare dollars spent), and nursing care facilities ( $\$ 143.1$ billion, 5.5\% of healthcare dollars spent). With healthcare costs continuing to rise, there is increased pressure to contain and reduce costs. One area of potential cost savings is reducing 30-day hospital readmissions.

\section{Types of Hospital Readmissions}

The American Hospital Association recognizes four types of hospital readmissions (2011):

- Planned readmissions related to the initial (referred to as the "index" admission): these are readmissions related to the index admission, such as a patient undergoing chemotherapy treatment may require planned hospital admissions to administer treatment or monitor progress. 
- Planned readmission not related to the index admission: these readmissions are planned but unrelated to the index admission, such as a total knee replacement following discharge for AMI.

- Unplanned readmission unrelated to the index admission: these are unplanned readmissions unrelated to the index admission, such as hospitalization for a car accident following CABG surgery.

- Unplanned readmission related to the index admission: these are unplanned readmissions related to the index admission, such as an infection at a surgical incision site.

It is the unplanned readmissions related to the index admission that CMS and the Medicare Payment Advisory Commission (MedPAC) have identified as an opportunity to reduce healthcare costs. In addition, MedPAC (2007), the American Hospital Association (2011), and Jencks, Williams, and Coleman (2009) estimated that up to 76\% of 30-day hospital readmissions are preventable and could save over $\$ 17$ billion in healthcare costs.

\section{Consequences of 30-Day Hospital Readmissions}

According to the MedPAC (2007), approximately 20\% of all Medicare discharges will result in a hospital readmission within 30 days of the index discharge. Of particular importance are three diagnoses being closely followed by CMS: AMI, PN, and HF. Beginning in FY 2013, CMS assessed penalties in the forms of reduced payments to hospitals if 30-day readmission rates for AMI, PN, and HF were above the established benchmarks. The United States Department of Health \& Human Services, along with 
CMS, used data from July 1, 2008, to June 30, 2011, in determining the benchmarks. Hospitals are evaluated based on data obtained from the previous three years. Thus, FY 2013 penalties were derived from readmission rates obtained from July 1, 2009, until June 30, 2012. The established 30-day readmission rate benchmarks for AMI, PN, and HF are $19.7 \%, 18.5 \%$, and $24.7 \%$, respectively. Additionally, beginning in 2014, CMS allowed accountable care organizations (ACO) to start distributing a portion of healthcare savings to its members as an incentive for providing high quality care to patients and reducing healthcare expenses. An ACO is a group of caregivers, comprised of physicians, hospitals, and other care providers who coordinate care with the intent of providing high-quality, cost-effective care (CMS, n.d.). A portion of the money that is saved as a result of reduced costs gets divided among hospitals and physicians, and serves as an incentive to establish an efficient, high-quality healthcare model.

The penalties for 30-day hospital readmission rates above the established benchmark are as follows:

- FY 2013: -1\% payment for all CMS patients based on readmission rates for AMI, PN, and CHF

- FY 2014: -2\% payment for all CMS patients based on readmission rates for AMI, PN, and CHF, CABG and chronic obstructive pulmonary disease (COPD), plus a $50-60 \%$ reduction in shared savings program by ACO

- FY 2015: -3\% payment for conditions listed in FY 2014, plus the likelihood of other diseases being added, plus a $50-60 \%$ reduction in shared savings program by ACO 


\section{Incidence of 30-Day Hospital Readmissions for AMI}

Currently, the national benchmark for 30 -day hospital readmissions is $19.7 \%$.

Dharmarajan et al. (2013) investigated causes for 30-day hospital readmissions for AMI, PN, and HF. The investigators reviewed all Medicare Fee-For-Services data from 30 different condition categories during 2007-2009 to consistently be able to determine readmission diagnoses with the following objectives:

- To determine the percentage of 30-day readmissions on each day following discharge

- To establish the primary causes for hospital readmissions during days 0-3; days $0-7$; days $0-15$; and days $0-30$ as well as the primary causes for hospital readmissions for consecutive days $(0-3 ; 4-7 ; 8-15 ; 16-30)$ following initial discharge

- To calculate the median time, in days, to hospital readmission

- To investigate any relationship between patient demographics (age, sex, race) and readmission diagnoses and timing

The study included all individuals age 65 and older who had a complete claims history one year prior to their hospitalization. Exclusion criteria included in-patient death, transfer to an acute care facility following discharge, discharge against medical advice, or discharge less than 30-days after enrolling in Medicare Fee-For-Services plan.

Nearly $68 \%$ of all 30 -day hospital readmissions for AMI occurred within 0-15 days of discharge, and over $30 \%$ occurred within 16-30 days of discharge. Nearly all (97.4\%) of patients readmitted were admitted once within 30 days; $2.4 \%$ were readmitted 
twice within 30 days; and $0.2 \%$ were admitted three or more times within 30 days of discharge. The five most common readmission diagnoses in the AMI cohort were, in order: HF, AMI, renal disorders, arrhythmias, and PN. These diagnoses accounted for $44.3 \%$ of the readmissions in the AMI cohort, whereas cardiovascular causes accounted for $53.4 \%$ of all AMI readmissions. The median time to rehospitalization in the AMI cohort was 10 days; however, the median time to rehospitalization for the top five diagnoses ranged from 9-11 days. There was no relationship between age, race, and sex and readmission diagnoses or timing.

In summary, many variables go into a 30-day hospital readmission following a discharge for AMI. Most readmissions occur within the first 15 days of discharge and are cardiac related; however, there appears to be no relationship between age, sex, race, and 30-day hospital readmissions.

\section{Incidence of 30-Day Hospital Readmissions for PCI}

The utilization of PCI as the treatment for intracoronary lesions has increased significantly in the past decade (D. Williams et al., 2000). The use of drug-eluting stents has improved patient outcomes and decreased the need for more invasive surgical interventions in the treatment of CAD.

Ricciardi et al. (2012) sought to examine the incidence of 30-day hospital readmissions following PCI as well as determine predictors for 30-day hospital readmissions. Patients $(n=10,965)$ from the Dynamic Registry were reviewed during 1999-2006 at 27 different hospitals. The Dynamic Registry initially involved 15 test centers throughout the United States, and has now expanded to include more hospitals. 
Patients receiving PCI at any of the test centers were enrolled into the registry. The purpose of the Dynamic Registry was to monitor clinical outcomes with PCI. Outcomes that were measured included length of stay (LOS), 30-day hospital readmission rates, and 30-day and 1-year mortality rates.

From 1999-2006, LOS and mortality significantly improved. Prior to 2004, no drug eluting stents were used; from 2004 until the completion of study, drug eluting stents were predominantly used. The 30 -day readmission rates ranged from $0-14.3 \%$ at the 27 sites, but averaged $4.6 \%$ over the length of the entire study. It was found that in 2004, 30-day hospital readmission rates did temporarily increase due to issues with the Cordis CYPHER ${ }^{\mathrm{TM}}$ Coronary Stent pending public notification of these issues by the Food \& Drug Administration (FDA). Patients who were readmitted within 30 days had a higher one year mortality rate than those who were not readmitted. Furthermore, technical aspects of performing PCI had no bearing on the likelihood for readmission. Readmission risks appeared to be related to patient demographic characteristics.

\section{Incidence of 30-Day Hospital Readmissions for CABG}

Because of the increasing use of PCI, CABG surgery is less common, and other modes of delivery of CABG are available now, such as totally endoscopic CABG surgery (TECAB).

Stewart et al. (2000) investigated the incidence of 30-day hospital readmissions at Beth Deaconess Medical Center in Boston, Massachusetts, and also sought to determine predictors of readmissions. 
During 1997, 503 patients underwent CABG surgery at Deaconess Medical Center. Those who died in the hospital $(n=16)$ or were still hospitalized at post-operative day $30(n=2)$ were excluded. The investigators contacted each patient on the $30^{\text {th }}$ post-operative day and asked if they had been hospitalized for any reason since their CABG surgery.

Seventy-six patients $(16 \%)$ were readmitted to the hospital within 30 days of discharge. The most common readmitted diagnoses were atrial fibrillation (22\%), saphenous vein graft wound infection (17\%), HF or angina (14\%) and other diagnoses, either encompassing multiple diagnoses or no definite diagnosis reason given (16\%). There was a significant, inverse relationship between LOS and readmissions. Only 13\% of patients with a LOS of less than seven days were readmitted, compared with $23 \%$ of patients with a LOS of greater than seven days who were readmitted.

In summary, atrial fibrillation, saphenous vein graft wound infection, HF, or angina appear to be the primary reasons for 30-day hospital readmissions following CABG surgery.

\section{Incidence of 30-Day Hospital Readmissions for STEMI}

Little research has been done to ascertain the causes of, and determine risk for, hospital readmissions following STEMI. Although STEMI is a form of AMI, there may be an increased risk for unplanned readmissions and mortality.

J. Brown, Conley, and Niles (2013) sought to establish 30-day hospital readmission rates following STEMI and to determine if any variables offered any predictors for unplanned readmissions. A total of 1,271 individuals who presented to 
Dartmouth-Hitchcock Medical Center with a confirmed diagnosis of STEMI between 2006-2011were retrospectively enrolled in the study. Readmission was defined as the time period from presentation to the medical center with STEMI to 30 days beyond the date of presentation. All readmissions were verified and reviewed by the researchers and the causes of readmission were documented in a database to determine readmission rates. Emergent readmissions and post-discharge death 30 days after STEMI were used in developing the readmission prediction model.

There were 109 emergent readmissions and 26 deaths within 30 days after STEMI for a 30 -day readmission rate of $11.7 \%$. There were 14 elective readmissions, which were not included in the 30-day readmission rate. The most common readmission diagnoses were ischemic heart disease $(43 \%, n=54)$, chest pain $(16 \%, n=20)$, gastrointestinal (GI) issues $(8 \%, n=10)$, and $\mathrm{HF}(7 \%, n=9)$.

In summary, patients who presented with STEMI had a nearly $12 \% 30$-day hospital readmission rate, with cardiac complications (ischemic heart disease and chest pain) being the most common readmitting diagnoses.

\section{Predicting 30-Day Hospital Readmissions}

CMS currently tracks 30-day hospital readmission rates for AMI, PN, and HF. In 2014, CABG and COPD were added as monitored diagnoses. It has become evident in light of the financial repercussion associated with unplanned readmissions for hospitals and physicians to be able to determine patients at risk for unplanned readmissions. 


\section{Predicting Readmission Risk for AMI}

In a retrospective study done by Kociol and colleagues (Kociol, 2011, Kociol et al., 2012) the strongest predictors for unplanned 30-day readmissions following AMI were:

- Baseline HR > 60 beats/minute (bpm): every 10-beat increase in baseline HR above $60 \mathrm{bpm}$ increased the risk for 30-day readmissions by $12 \%$

- Age: every year over age 55 increased the risk for 30 -day readmissions by $2 \%$

- Being hospitalized in the United States increased the likelihood for 30-day readmissions by $53 \%$ compared to the rest of the world

- Only Denmark and Sweden have higher 30-day readmission rates than the United States

- Patients with a history of hypertension were $37 \%$ more likely to be readmitted within 30 days than patients with normal BP

- Patients with a previous history of CAD were $44 \%$ more likely to be readmitted within 30 days than patients with no prior history of CAD

- When LOS is $>6$ days, there is a $69 \%$ increase in the risk for unplanned 30-day readmissions

- There is a $17 \%$ decrease in the risk for 30 -day readmissions for each day below the median LOS that the hospitalization lasts

- Caucasians have a $48 \%$ lower risk for 30-day readmissions than any other race or ethnic group 
- Patients with a history of COPD have a 54\% greater risk for unplanned readmissions within 30 days of discharge

- When patients develop atrial fibrillation during their hospitalization, the risk for 30-day readmission increases by $54 \%$

- If the AMI is a non-inferior MI (i.e., septal, lateral, and/or anterior MI), there is a $24 \%$ greater likelihood for hospital readmission within 30 days of discharge

In summary, there appear to be systemic variables in place such as LOS, location of hospitalization (i.e., country), as well as patient demographic and comorbidities that offer predictive value for the risk for 30-day hospital readmissions such as age, race, prior history for CAD, type of AMI, atrial fibrillation, and other risk factors.

\section{Predicting Readmission Risk for STEMI}

One of the objectives of the study by J. Brown et al. (2013) was to determine any variables that could increase the likelihood for unplanned, 30-day hospital readmissions. Of the many factors investigated, Brown et al. identified several factors that were predictive of not only 30-day hospital readmission, but death as well. These variable were listed by category:

- Patient comorbidities:

Age $\geq 80: 2.25$ times higher risk for readmission or death

○ Diabetes: 1.9 time higher risk for readmission or death

○ Hypercholesterolemia: 33\% lower risk for readmission or death

- Disease and presentation at hospital: 
○ Chest pain upon presentation: 3.37 times higher risk for readmission or death

○ Cardiac arrest upon arrival or during hospital admission: 3.02 times higher risk for readmission or death

○ Triple vessel disease: 1.95 times higher risk for readmission or death

○ Transfer patient: $44 \%$ lower risk for readmission or death

○ Clopidogrel given during PCI procedure: $31 \%$ lower risk for readmission or death

- Inpatient outcomes:

New onset of HF: 2.15 times higher risk for readmission or death

- Discharge medications:

○ Core measure medications (beta-blocker, ACE or ARB, aspirin, statin): $33 \%$ lower risk for readmission or death

In summary, patient demographics such as age or diabetes are predictive of the risk for readmission or death. Additionally, patients with a new onset of HF during the hospitalization, triple vessel disease or who suffered cardiac arrest at the time or during their hospitalization were more likely to be readmitted or die. Patients who transferred from other facilities, were diagnosed with hypercholesterolemia, received clopidogrel during their PCI, or were discharged with beta-blockers, ACE or ARB inhibitors, statins, and aspirin were less likely to be readmitted or die. 


\section{Predicting Readmission Risk for PCI}

When the study by Ricciardi et al. (2012) began, bare metal stents (BMS) and balloon angioplasties were the two primary forms of PCI. During the latter part of the study, drug eluting stents (DES) were used primarily in favor of BMS and other forms of PCI. The following factors were predictive of 30-day readmissions:

- Sex: women were $39 \%$ more likely to be readmitted than men

- New onset of HF: HF occurring during hospitalization increased risk for readmission by $41 \%$

- Other comorbidities: patients with multiple comorbidities (i.e., smoking, diabetes, dyslipidemia, etc.) were $31 \%$ more likely to be readmitted

- Reason for PCI:

- Patients who received PCI for unstable angina were $66 \%$ more likely to be readmitted

- Patients who received PCI because of AMI were 52\% more likely to be readmitted

- Number of significant lesions: patients with $>3.1$ significant lesions were $15 \%$ more likely to be readmitted

- Emergency PCI: patients who required emergent PCI were 53\% more likely to be readmitted

- The number of lesions treated decreased the risk for readmission by $16 \%$ In summary, there were no procedural characteristics that increased the likelihood for 30-day readmissions. Patient characteristics were highly predictive of readmissions 
and included female sex, new onset of HF, comorbidities, reason for PCI, and the number of significant lesions. Conversely, the more lesions that were treated by PCI decreased the risk for 30-day hospitalizations.

\section{Predicting Readmission Risk for CABG}

Stewart et al. (2000) identified reasons for 30-day rehospitalizations and predicted the risk for readmissions as well. The main predictors for readmission were:

- Sex: women were more than twice as likely to be readmitted as men

- History of diabetes: diabetics were $76 \%$ more likely to be readmitted than non-diabetics

- Post-operative AF: patients with post-operative AF were $77 \%$ more likely to be readmitted than patients not in AF

In summary, women were much more likely to be readmitted than men, and also had higher incidence of post-surgical infection, one of the leading causes for readmission. Diabetics and patients who developed AF were at higher risk for readmission. Interestingly, HF and PVD were not significant predictors for readmission, although HF was nearing statistical significance $(p=0.09)$.

\section{Private Insurance Versus Public Insurance}

Jiang and Wier (2010), as part of the Healthcare Cost and Utilization Project (H-CUP), compared readmission rates for non-elderly individuals with Medicare and Medicaid with individuals with private insurance during 2007. Medicaid non-obstetric readmission data from 10 states: Arkansas, Arizona, Florida, Hawaii, Montana, Nebraska, New Hampshire, New York, South Carolina, and Tennessee, were compared with private 
insurance readmission data. Obstetric data was obtained from three states: Arizona, Nebraska, and South Carolina.

Readmission rates were significantly higher at 7, 14, and 30 days for Medicaid insurance compared with private insurance with all patients, the exception being obstetric patients aged 0-20. All adults aged 21-64 with Medicaid had significantly higher readmission rates at 7, 14, and 30 days compared to private insurance across all age categories, regardless of sex.

Additionally, those with Medicaid had consistently higher readmission rates, regardless of the number of comorbidities $(0,1,2$, or $3+)$ than those who were privately insured. For some specific comorbidities (anemia, diabetes, chronic lung disease, substance abuse, and mental disorders), readmission rates were also significantly higher among Medicaid recipients compared to those with private insurance.

Wier, Barrett, Steiner, and Jiang (2011) compiled 2008 readmission data from 15 states: Arizona, California, Florida, Hawaii, Louisiana, Maine, Missouri, Nebraska, New Hampshire, New York, South Carolina, Tennessee, Utah, Virginia, and Washington, and compared readmission rates for private insurance with Medicare and Medicaid readmission rates. Medicare patients aged $>65$ years had an overall readmission rate of $19 \%$, whereas Medicare patients aged $18-64$ had a $24.1 \%$ readmission rate in 2008 . The non-maternal readmission rates in 2008 for Medicaid, private insurance and uninsured for ages $1-17$ were $12 \%, 11 \%$, and $7 \%$, respectively. The non-maternal readmission rates in 2008 for Medicaid, private insurance, and uninsured for ages 18-44 were 20.8\%, 10.1\%, and $11.2 \%$, respectively. The non-maternal readmission rates in 2008 for Medicaid, 
private insurance, and uninsured for ages $45-64$ were $24.4 \%, 11.9 \%$, and $14.4 \%$, respectively.

Jencks et al. (2009) investigated all patients enrolled in the Medicare Fee-For-Services (FFS) program during 2003-2004. Of the 11,855,702 Medicare patients hospitalized in 2003-2004, 19.6\% were readmitted within 30 days, and 34\% were readmitted within three months. Over half of the patients discharged following a 30-day readmission were not seen by any physician. Further, $51.5 \%$ of patients admitted with a surgical diagnosis were either readmitted to the hospital or died within one year of the index hospitalization, and $67.1 \%$ of patients admitted for medical reasons either died or were readmitted within one year of the index discharge.

There were many predictors for readmission. Males were more likely to be readmitted than women, and African Americans were more likely to be readmitted than any other race. Low-income patients, especially patients who received Supplemental Security Income (SSI), were more likely to be readmitted than higher income counterparts. There was a positive correlation between the number of previous admissions within the 12 months and readmission risk. Patients who had been admitted to the hospital $\geq 3$ times in the past 12 months were 2.5 times more likely to be readmitted than someone who had not been admitted. LOS was positively correlated with increased risk, as patients who were hospitalized twice as long as expected based on the diagnosis related group (DRG) were more likely to be rehospitalized.

In summary, individuals with private insurance have lower readmission rates than those with public (Medicare/Medicaid) insurance. Uninsured individuals tend to have 
higher readmission rates than those who are privately insured. Some of the disparity in readmission rates can be attributed to patient demographics, whereas other factors such as the number of comorbidities an individual has is also a strong predictor.

\section{The Relationship Between LOS and 30-Day Readmission Rates}

It has been assumed that as LOS is reduced, the risk for unplanned 30-day hospital readmissions increases. Today, there are increasing financial pressures for hospitals to reduce LOS because of the bundling of charges based on DRG. Patients at times may feel overwhelmed with new diagnoses. In addition, they may not receive appropriate education or instruction at the time of discharge. With hospitals tightly managing staff to patient ratios as a means of controlling overhead costs, the perception can be that the level of care is decreasing. Hospitals feel they are being penalized twice: first, if they fail to discharge a patient in a timely fashion based on DRG, and second, if the patient is readmitted within 30 days of discharge.

Kaboli et al. (2012) retrospectively analyzed data from 129 Veterans Affairs (VA) hospitals in the United States from 1998-2010. A subset of over 4 million patients with either HF or COPD as a chronic disease or AMI, PN, or GI hemorrhage as an acute disease process was investigated. The two primary outcome variables studied were LOS and 30-day readmission rates, and a secondary outcome measure was mortality rates. The authors hypothesized that decreasing LOS would lead to increased 30-day readmission rates and increased risk for mortality.

When adjusting for variables such as age, sex, socioeconomic status, marital status, comorbidities, and mode of entrance into the VA hospital, LOS significantly 
decreased by nearly 2 days, from 6.33 days to 4.40 days for HF from 1998-2010, and AMI significantly decreased by nearly 3 days, from 6.63 days to 3.78 days (Kaboli et al., 2012). The other diseases also reported significant reductions in LOS; the overall LOS was reduced on average by $27 \%$, or 1.46 days, from 1998-2010.

When the same variables were accounted for, there was a significant decrease in 30-day readmission rates for 2003-2010 compared with 1998-1999 for HF (an average of 1.4 percentage points lower by 2010). There was also a significant decrease in 30-day readmission rates for AMI in the years 2001-2010 compared to 1998-1999 (an average of 2.8 percentage points lower by 2010 ). Mortality rates significantly decreased by $25 \%$ at 30 days post-discharge, and by $18 \%$ at 90 days post-discharge. Interestingly, patients who were discharged 1 day earlier than the average LOS had a $6 \%$ higher risk for 30 -day readmission, and patients who were discharged 1 day later than the average LOS had a 3\% higher risk for 30-day readmission (Kaboli et al., 2012).

In summary, LOS, 30-day readmission rates, and mortality decreased during the time period of 1998-2010. Some of the improvements can be attributed to process improvements and efficiencies in delivery of care. Additionally, improved medical therapies likely contributed to the improved outcomes. Although this study was limited to VA hospitals (Kaboli et al., 2012), it was the first study to compare LOS and 30-day readmission rates, and to determine that reducing LOS did not have a significant impact on 30-day readmission rates. 


\section{0-Day Episodes of Care as a Quality Metric}

With the financial implications of unplanned 30-day readmission rates and the increased emphasis on reducing LOS and readmissions, there is debate as to whether 30-day readmission rates are the appropriate metric to measure quality of care. While CMS has emphasized 30-day readmission rates as a quality indicator, another indicator that may better portray quality and utilization of healthcare resources is the 30-day episode of care (EOC). EOC is defined as the total number of inpatient days for an index hospitalization during a 30-day period (Kociol et al., 2013).

Kociol et al. (2013) looked retrospectively at LOS and EOC and compared them with 30-day readmission rates and mortality rates to determine if any correlation existed between the variables. The data were collected using the AHA Get With The Guidelines-Heart Failure (GWTG-HF) quality improvement registry during January 2005-December 2006. A total of 17,387 patients from 149 hospitals participating in the AHA GWTG-HF registry were studied. The hospitals were divided into four quadrants (1-4) based on hospital-risk readmission rate, mortality rate, LOS, and EOC.

There was no relationship between LOS and 30-day readmission rates. There was a statistically significant relationship between EOC and 30-day readmissions, although the correlation was modest $(r=.26)$. Hospitals in the lowest quartile of LOS had significantly lower mortality rates than hospitals in the highest quartile of LOS. Additionally, hospitals in the lowest 2 quartiles of EOC had significantly lower mortality rates than hospitals in the highest quartile of EOC. Additionally, there was no relationship between 30-day readmission rates and mortality rates (Kociol et al., 2013). 
These findings suggest that 30-day readmission rates may not be the ideal metric to measure quality. Although this study was limited to HF, CMS is tracking readmission rates for chronic diseases, so this study may be the basis for reevaluating which metric(s) are and should be used to measure quality. Interestingly, hospitals that were $100 \%$ compliant with CMS performance measures, such as core measure medications, had higher readmission rates than less compliant hospitals. Hospitals that had lower EOC had significantly lower mortality rates; 30 -day readmission rates were not correlated with mortality rates.

In summary, 30-day readmission rates are not related to mortality rates for HF, but 30-day EOC are significantly correlated with mortality rates. These findings are particularly interesting given the emphasis and accountability (expressed in financial penalties) that CMS has placed on 30-day readmission rates for HF. Because of these findings, it is possible that in the future, CMS may look at EOC as the ideal quality and efficiency metric rather than 30-day readmission rates.

\section{Preventing 30-Day Hospital Readmissions}

Jack et al. (2009) designed a novel program whose sole purpose was to reduce 30-day readmissions and emergency department (ED) visits following hospital discharge. Although there are many methods to discharge and educate patients prior to discharge, no method of intervention has been studied that showed a reduction in 30-day readmission rates.

The program, called reengineered discharge (RED), involves a total of 11 components, encompassing three phases: the in-hospital phase, the post-discharge phase, 
and the pharmacist post-discharge intervention (Jack et al., 2009). The in-hospital component was performed by an RN known as a discharge advocate (DA). The inhospital interventions consisted of:

- Educating patients about their diagnoses during their hospitalization

- Make follow-up appointments for relevant outpatient testing, outpatient services and physician appoints

- Reinforce the importance of maintaining these appointments and discuss any barriers to keeping the appointments (i.e., transportation, etc.)

- Discuss any pending inpatient tests and determine who will review the results with the patient

- Organize all discharge services and reinforce the importance of keeping all appointments and services

- Organize medication reconciliation, including which medications to take, how to take them, and the mode of action for each medication

- Review how the patient will obtain all medications

- Organize discharge plan based on national guidelines and critical pathways

- Review plans if issues arise: give patient physician phone number(s), what circumstances determine an emergency and when they should contact physician(s) or report to the ED

- Send discharge summary to all relevant physicians, specialists and providers 
- Summarize the reason for hospitalization, diagnoses, findings, tests and procedures performed, discharge condition, medication reconciliation, findings from specialty consults, lab values, and test results

- Determine the patient's level of understanding by having the patient explain the discharge plan in his/her own words

The after-hospital care plan (AHCP) was created by the DA, and consisted of a written discharge plan provided to the patient containing the admitting diagnosis; medication reconciliation, including how to take each medication; contact information for physician(s); and a calendar with dates and times for all appointments (Jack et al., 2009).

A pharmacist would then contact patients 2-4 days post-discharge to again review and reinforce the discharge care plan, and to discuss any issues with the care plan, including medications. Any issues were then reviewed with the DA or the patient's primary care physician (PCP).

In this study (Jack et al., 2009), a total of 749 patients who were admitted to Boston Medical Center were randomized to either an intervention group that received the RED program or a control group that received usual care. The primary endpoint for the study was utilization of hospital within 30 days of discharge, either by readmission or by ED visit. Secondary endpoints included patient preparedness upon hospital discharge, rate of PCP visits, and understanding of their discharge diagnosis.

Participants in the intervention group had significantly fewer hospital utilizations (either readmissions or ED visits), averaging 0.314 visits/person/month, compared with 
0.451 visits/person/month in the usual care group. Participants in the intervention group were nearly $31 \%$ less likely to be readmitted to the hospital or visit the ED than those in the usual care group. Additionally, participants in the intervention group were significantly more knowledgeable about their discharge diagnosis (79\% vs. 70\%), had significantly higher follow-up rates with PCP ( $62 \%$ vs. $44 \%)$, and were more prepared to be discharged from the hospital (65\% vs. 55\%) compared with the usual care group (Jack et al., 2009).

Participants in the intervention group also noted a nearly $34 \%$ reduction in healthcare costs, equal to approximately $\$ 412 /$ person. This included reduced costs of ED visits, hospitalizations, and follow-up PCP visits. Although the intervention group had higher costs associated with PCP visits (because of improved compliance), the overall cost reduction for the intervention group was $\$ 149,995$.

The study (Jack et al., 2009) was limited to one hospital and was limited by the number of participants that could be enrolled daily. Additionally, the demographics of the participants revealed a generally healthier population because patients with multiple comorbidities were excluded, so these findings may not be generalized to all population groups. Cost savings did not include the cost of providing the DA and the pharmacist, which consisted of a 0.5 full-time equivalent (FTE) for the DA and a 0.15 FTE for the pharmacist.

In summary, these findings suggest that allocating resources to patient education during the hospitalization, involving patients in the discharging process, and organizing post-hospitalization care may reduce hospital utilization upon discharge. It also appears 
that devoting resources to planning patient discharge and education may have additional economic benefits for patients and reduce healthcare costs. Reducing hospital utilizations could reflect in improved quality measures that are publicly reported and may be more attractive to insurance providers (Jack et al., 2009).

\section{Cardiac Rehabilitation Reducing Hospitalizations}

Hospital readmissions are not just an issue in the United States but also a world-wide problem. In Australia, it is estimated that hospital readmissions from CVD cost approximately $\$ 2.75$ billion annually (Canyon \& Meshgin, 2008). Canyon and Meshgin hypothesized that participating in a cardiac rehabilitation would reduce long-term rehospitalizations, and potentially reduce healthcare costs.

During 2000-2005, 954 patients were enrolled in the HeartBeat ${ }^{\mathrm{TM}}$ program, a CR program that patients attended one day per week, for seven weeks. The CR program consisted of a one-hour exercise session followed by a one-hour intense education class focusing on topics such as lifestyle modification and nutrition. To qualify for the study, participants had to attend at least five sessions. A total of 110 participants attended at least five sessions, and 198 participants did not attend any sessions and served as a control group. The primary endpoint of the study was a non-elective readmission for any cardiac reason in the 12 months following the completion of the HeartBeat ${ }^{\mathrm{TM}}$ program.

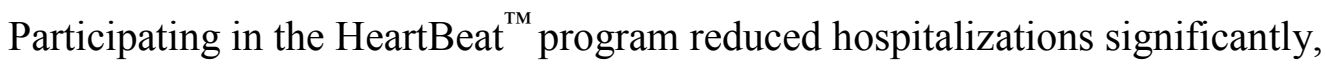
and also significantly reduced LOS when participants were hospitalized for cardiac reasons. Those who deferred participating in the HeartBeat ${ }^{\mathrm{TM}}$ program were more than three times more likely to be hospitalized for cardiac reasons within 12 months of their 
index hospitalization. Participants in this study had the choice to enter the HeartBeat ${ }^{\mathrm{TM}}$ program, which may have been a potential source of bias. Disease severity was not evaluated, which may have impacted motivation to participate.

In summary, it appears that CR may decrease 12-month hospital readmissions for cardiac reasons. Participating in CR may significantly reduce the likelihood for future readmissions because of the strong evidence supporting secondary prevention of cardiovascular risk factors and CR programs.

\section{Cardiac Rehabilitation Beginning at the Bedside}

CR programs have historically had both very low referral and participation rates (AHA GWTG, 2009; Ades et al., 1992; Cortes \& Arthur, 2006; Lavie et al., 2009; Thomas, 2007; Thomas et al., 1996; Thomas, Witt, Lopez-Jimenez, King, \& Squires, 2005) for a variety of reasons. Institutional and physician support is vital to not only referring patients, but also getting the patients to participate in CR. Perhaps the best way to increase referral rates and admissions into CR programs is to institute a bedside CR program.

Tiller, Leger-Caldwell, O’Farrell, Pipe and Mark (2013) instituted an automatic referral process for CR at The University of Ottawa Heart Institute. Prior to 2006, referral rates for CR were approximately $26.7 \%$ and participation rates were 15.5\%-19.7\%, because CR referrals were physician-driven. The physician-based referral process had very labile referral rates. Beginning in 2006, CR referrals were no longer physician-driven; RNs, ward clerks, and other healthcare professionals could refer patients to $\mathrm{CR}$ based on clinical diagnosis and need. A referral would be sent to the CR 
department, and a letter was mailed to the patient asking them to participate in CR. An internal review showed the process was still flawed and needed further revisions. In 2008, The University of Ottawa Heart Institute began a bedside RN CR referral process to increase referral and participation rates.

In 2003-2004, only $26.7 \%$ of eligible patients were referred to CR, and only $15.5 \%$ of eligible patients participated in CR. In 2008-2009, after utilizing bedside CR referrals, $79 \%$ of eligible patients were referred to CR, with $32.6 \%$ of eligible patients participating in $\mathrm{CR}$. These results were achieved utilizing 1.5 RN full-time equivalents (FTE).

CR typically does begin at the bedside. Many patients admitted with an eligible diagnosis may not be aware they are eligible to participate in CR. Perhaps the best opportunity to initiate $\mathrm{CR}$ is during the hospitalization, prior to discharge. The referral can be placed and CR intake can be planned at that time. Although this process has been shown to not only increase referral rates but participation rates as well, fewer programs are instituting this practice. Because of the bundling of charges associated with DRG, many inpatient $\mathrm{CR}$ visits, called Phase I or inpatient $\mathrm{CR}$, are not reimbursed, and therefore are no longer done.

In summary, CR initiated at the bedside during hospitalization has been shown to significantly increase both referral rates and participation rates in outpatient CR programs. Economic and institutional factors influence how prevalent bedside CR programs are. 


\section{Problem/Rationale}

Changes in Medicare's Fee-For-Services program as part of the PPACA (2010) now penalize hospitals for 30-day readmission rates above 19.7\% for AMI (MedPAC, 2007). Hospitals with unplanned 30 -day readmission rates above the $19.7 \%$ threshold face a $1 \%$ penalty on CMS reimbursements in 2013, with escalating penalties beginning in 2014 (MedPAC, 2007; PPACA, 2010). While CMS acknowledges that some readmissions are unavoidable, and in some cases, even planned, it is the unplanned readmissions related to the index admission that CMS is targeting. The United States Subcommittee on Healthcare Financing (MedPAC, 2007) estimates $76 \%$ of readmissions are preventable and that excessive, unplanned 30-day readmissions cost up to $\$ 17$ billion.

Several studies have attempted to predict readmission risk for STEMI, AMI, PCI, and CABG (J. Brown et al., 2013; Dharmarajan et al., 2013; Kociol, 2011; Kociol et al., 2012; Ricciardi et al., 2012; Stewart et al., 2000), each unique diagnosis having predictors for readmission. Although there appears to be no homogenous predictors for unplanned 30-day readmissions, there is increased emphasis on secondary prevention as a method to reduce 30-day readmission rates.

Evidence has shown that CR is a vastly underutilized service for secondary prevention of CVD (AHA GWTC, 2009; Ades et al., 1992; Cortes \& Arthur, 2006; Lavie et al., 2009; Thomas, 2007; Thomas et al., 1996; Thomas et al., 2005) despite the numerous documented benefits, including decreased risk for cardiovascular and all-risk mortality as well as morbidity (Artham et al., 2008; Lavie et al., 2008; Squires et al., 1990; Squires \& Hamm, 2006; Thomas et al., 2007; Williams et al., 2006). In addition, 
CR participation has been shown to reduce readmission rates at 12 months post-discharge (Canyon \& Meshgin, 2008), and to reduce the incidence of cardiovascular events and hospitalizations as long as five years after completing CR (Plüss et al., 2011).

There is very little research that examines the effects of CR on readmission rates, specifically, unplanned 30-day readmission rates. If CR was shown to reduce unplanned 30-day readmission rates, it could lead to increased access, referral, and participation in CR programs not only in the United States, but around the world.

\section{Purpose}

The purpose of this study was to determine if unplanned 30-day readmission rates were lower among patients who participated in a monitored, Phase II CR program, than patients who did not participate in a Phase II CR program. The primary outcome for this study was 30-day readmission rates for patients who participated in Phase II CR and for those who did not participate in CR. An area of particular interest is attempting to determine what factors may predict non-participation in $\mathrm{CR}$ and what factors are predictive for 30-day rehospitalizations. Demographic factors such as age, sex, race, type of insurance (private vs. public), comorbidities, number and type of medications were evaluated to determine what relationship each has with 30 -day readmission rates. The following hypotheses were investigated:

Hypothesis 1: Patients who participate in a Phase II CR program will have lower unplanned 30-day readmission rates than patients who do not participate in CR. 
Hypothesis 2: Patients who have private insurance will have lower 30-day unplanned readmission rates than patients with publicly funded insurance (i.e., Medicare, Medicaid).

Hypothesis 3: Men will have higher 30-day unplanned readmission rates than women.

Hypothesis 4: Economic factors (i.e., employment status, type of health insurance) will be a significant predictor of participation in CR. 


\section{CHAPTER III}

\section{METHOD}

\section{Study Design}

This study investigated the effect that participating in a CR program may have on unplanned 30-day readmission rates. This is a retrospective analysis that evaluated if 30-day readmission rates were lower in a patient population that participated in CR compared with patients who did not participate in CR. A priori power analysis using G*Power 3.0 was conducted to determine the necessary sample size needed to show a significant difference in 30-day readmission between patients who participated in a CR program and those who did not. Results of a power analysis for the chi-square test indicated that a minimum sample size of 90 participants (45 in each arm) was required to observe a medium effect size $(\mathrm{w}=.30)$ at $80 \%$ power when the significance level is set at $p \leq .05$. Additionally, patient demographics, medical history, and comorbidities were documented to determine if there were factors that may predict non-participation in CR and if non-participation in CR increases the likelihood for readmission. All patients in the sample were referred to CR; patients who deferred CR served as a control group, and patients who participated in CR served as the treatment group. The dependent variable in this study was the calculated 30-day readmission rate, and the independent variables were participation status (participant or non-participant) in CR, demographic characteristics (i.e., age, marital status, race/ethnicity and gender), and comorbidities that could predict the risk for readmission. 


\section{Participants}

The current study consisted of 90 persons who were referred to a CR program during the timeframe of January 2012-June 2013 with the following diagnoses: MI, PCI (with or without stenting), CABG surgery, valve surgery (repair and/or replacement), HF, and stable angina pectoris. This is a retrospective study that compared unplanned 30-, 60-, and 180-day readmission rates in patients that participated in, and completed, $\mathrm{CR}$ and patients who did not participate in CR.

Participants were retrospectively recruited from Akron General Medical Center, a 376 bed, not-for-profit hospital in Akron, Ohio. Akron General Medical Center is a teaching hospital affiliated with several medical schools in and around Northeast Ohio. The participants in the prospective study reflect all patients who were eligible to receive CR during the timeframe of January 1, 2012-June 30, 2013.

The patients' index admission and secondary diagnosis (if applicable) were noted, and through retrospective analysis of medical records utilizing MidasPlus, Inc.

(Milliman, Inc., copyright 1984-2013), a database that records hospital discharges based on DRG, and ChartMaxx (version 5.60.53, Medplus, Inc. copyright 2008), an electronic medical record (EMR) that records hospital utilization (i.e., hospitalizations, outpatient tests and procedures, and ED visits), hospital utilization, including hospital readmissions, were documented.

Because the study is a retrospective analysis, no participant consent was obtained. The Institutional Review Boards (IRB) at Akron General Medical Center and Kent State University approved the study. Readmission rates were adjusted to account for the 
confound of patients with contraindications for participation in CR. To be eligible for participation in the study, patients needed a qualifying diagnosis for referral to CR: MI, $\mathrm{PCI}, \mathrm{PCI} /$ stent, angina pectoris, CABG surgery, valve repair or replacement, or HF. Participant demographics are shown in Table 1.

Table 1

Demographic and Readmission Data For Participants and Non-Participants

\begin{tabular}{lcc}
\hline & Participants & Non-participants \\
\hline Men & 36 & 29 \\
Women & 9 & 16 \\
Age & $65.8 \pm 11.17$ & $64 \pm 8.89$ \\
Readmit at 30 days & 3 & 9 \\
Readmit at 60 days & 1 & 4 \\
Readmit at 180 days & 1 & 8 \\
Total \# readmissions & 5 & 21 \\
Mean \# readmissions & $.1111 \pm .3178$ & $.5556 \pm 1.099$ \\
STO at 30 days & 1 & 3 \\
STO at 60 days & 1 & 2 \\
STO at 180 days & 7 & $1.08 \pm 1.12$ \\
\# lesions treated & $2.17 \pm 1.65$ & \\
\hline
\end{tabular}

Expressed as means $\pm \mathrm{SD}$. 


\section{Measures}

\section{Readmission Rates}

Unplanned 30-day readmission rates were calculated, beginning 30 days from the date of hospital discharge, based on discharge information from the EMR for the index admission on participants and non-participants in CR. All cause readmission rates, which include planned and unplanned admissions, were calculated, as were unplanned readmission rates related to the index admission.

\section{Readmission Risk}

Participant demographic data, medical history, and comorbidities were evaluated to determine if any variables were predictive in increasing the risk for unplanned 30-day readmissions.

\section{Non-Participation Risk}

Review of demographic data, including history and physical, medical history, and comorbidities were collected and analyzed to determine if there were factors that predict non-participation in $\mathrm{CR}$.

\section{Demographic and Medical History}

Demographic characteristics and history and physical were collected through an electronic medical record (ChartMaxx, version 5.60.53, Medplus, Inc., copyright 2008) and verified through the participant's medical history.

\section{Procedures}

The Institution Review Boards from Akron General Medical Center and Kent State University approved the study procedures. No participant consent was required 
because of the retrospective study format. Data were stored at Akron General Medical Center. No protected health information (PHI) such as name, address, phone number, medical record number, or account number was used during data collection and no protected health information or raw data was taken from the medical center. Hospital discharge information was collected during the months of January 2012-June 2013 for diagnoses of unstable angina, PCI, PCI/stent, MI, CABG, valve surgery, or HF. All patients during this discharge period were referred to CR unless medically contraindicated, and all patients had the option to enroll in CR following their index hospitalization. Retrospectively, individuals who participated in CR were placed in the treatment group, and non-participants were placed in the control group. Thirty-day readmission rates were calculated for each group to determine if participating in CR was associated with lower hospital readmission rates 30 days post-hospitalization.

\section{Statistical Analyses}

All statistical analyses were performed using SPSS ${ }^{\complement}$ Version 22.0. The experimental variables consisted of hospital readmission and short-term observation rates at 30,60, and 180 days, relative risk ratios for readmission, STO, and non-participation in $\mathrm{CR}$, as well as predictors for readmission, STO, and participation status. Some variables used to determine risk included age, gender, race/ethnicity, marital status, number of significant lesions at the time of hospitalization, number of lesions treated, history of COPD, history of diabetes mellitus (DM), fasting blood glucose levels, tobacco use, history of hypertension, prior cardiac event, history of stress/depression/anxiety, sedentary lifestyle, BMI, participation status in CR, primary and secondary diagnoses. 
The control variables for the covariance analyses were age, sex, ethnicity, number and type of comorbidities, and type of insurance. Means and standard deviations (SD) were computed on all dependent variables. A Mann-Whitney U non-parametric test was utilized to compare readmission and short-term observation (STO) rates for participants and non-participants in CR for 3 time points: 30, 60, and 180 days. A 2-tailed independent $t$-test determined if the total number of lesions differed between CR participants and non-participants. An independent $t$-test determined if the number of readmissions differed by participation status. The criteria for statistical significance or alpha level was set at $p<0.05$ for all tests. Readmission rates were calculated for the entire cohort by dividing the number of patients admitted within 30 days by the total sample. Stepwise regression was done to determine if factors pertaining to demographic information or health history, combined with condition, served as predictors for readmission, short-term observation, and participation in CR. Relative risk analysis, using hazard ratios and confidence intervals (CI) of 95\% were assessed to determine if participation in CR decreases the risk for readmissions or STO. 


\title{
CHAPTER IV
}

$\mathbf{1}^{\text {st }}$ MANUSCRIPT

\section{The Effect of Cardiac Rehabilitation on 30-, 60-, and 180-Day Unplanned Hospital Readmission and Short-Term Observation Rates}

\begin{abstract}
Hospitals are facing financial penalties for excessive 30-day readmission rates for various chronic diseases. Thirty-day readmission rates are now considered a quality indicator for hospital performance, and previous research suggests that up to $76 \%$ of 30-day readmissions could be prevented. The purpose of this study was to compare 30-, 60-, and 180-day readmission and short-term observation (STO) rates for participants and non-participants in a Phase II cardiac rehabilitation program. Ninety subjects (participants $n=45$; non-participants $n=45$ ) retrospectively participated in the study. All subjects were referred to Phase II cardiac rehabilitation (CR) following a qualifying cardiac diagnosis, and given the option to participate. Thirty-day readmission rates approached statistical significance and were higher for non-participants than participants (20\% vs. $6.7 \%, p=.064)$. Sixty-day readmission rates were higher for non-participants than participants $(8.9 \%$ vs. $2.2 \%, p=.170)$ but were not statistically significant. One hundred eighty-day readmission rates were significantly higher for non-participants than participants $(17.8 \%$ vs. $2.2 \%, p=.014)$. Thirty- and 60 -day STO rates were slightly higher for non-participants than participants $(6.7 \%$ vs. $2.2 \%, p=.309 ; 4.4 \%$ vs. $2.2 \%, p=$ .559) but were not statistically significant. One hundred eighty-day STO rates were statistically higher for participants than non-participants $(15.6 \%$ vs. $2.2 \%, p=.027)$.
\end{abstract}


Participating in Phase II cardiac rehabilitation appears to decrease the likelihood for readmission at 180 days. STO rates were higher at 180 days for Phase II cardiac rehabilitation participants, perhaps indicating a need for increased monitoring without requiring rehospitalization.

\section{Introduction}

Healthcare is a \$2.6 trillion industry (Centers for Medicare and Medicaid Services [CMS], 2012), with costs continuing to increase yearly. With healthcare costs continuing to rise, there is increased pressure to contain and reduce costs. One area of potential cost savings is through 30-day hospital readmissions, particularly unplanned hospital readmissions related to the index admission. It is the unplanned readmissions related to the index admission that the Centers for Medicare and Medicaid Services (CMS) and the Medicare Payment Advisory Commission (MedPAC) have identified as an opportunity to reduce healthcare costs. In addition, MedPAC (2007), the American Hospital Association (2011), and Jencks et al. (2009) estimated that up to $76 \%$ of 30 -day hospital readmissions are preventable and could save over $\$ 17$ billion in healthcare costs.

Prior research has investigated causes for, and predictors for, unplanned hospital readmission for acute myocardial infarction and ST elevation myocardial infarction (J. Brown et al., 2013; Dharmarajan et al., 2013; Kociol, 2011; Kociol et al., 2012), percutaneous coronary intervention (Ricciardi et al., 2012), and coronary artery bypass graft surgery (Stewart et al., 2000). In short, there are variables that serve as predictors for readmission. These variables fall into three categories: patient characteristics (i.e., age, race, gender, insurance), patient comorbidities (i.e., diabetes, atrial fibrillation, prior 
history of coronary artery disease), as well as systemic variables (i.e., length of stay, location of hospitalization).

Canyon and Meshgin (2008) determined that participating in cardiac rehabilitation (CR) may prevent hospitalizations 12 months post-index hospitalization, reduce healthcare costs, and reduce length of stay (LOS) when individuals were hospitalized for cardiac reasons. Despite the many benefits of cardiac rehabilitation (CR), it is a vastly underutilized service. According to the American Heart Association Get With the Guidelines (AHA GWTG 2009), only 56\% of all eligible patients were referred to CR. Of patients with AMI 53\% were referred, 58\% of patients with percutaneous coronary intervention (PCI) were referred, and $74 \%$ of patients with coronary artery bypass graft $(\mathrm{CABG})$ surgery were referred. There is an $18.7 \%$ attendance rate in CR programs in the United States (Lavie et al., 2009), consistent with attendance rates worldwide (Neubeck et al., 2011).

A better understanding of the interaction between CR and 30-, 60-, and 180-day readmission rates could prove beneficial as hospitals look to reduce readmission rates. If CR did affect readmission rates, the result could be increased referral and participation rates. The purpose of this study was to retrospectively investigate the effects of participating in an outpatient Phase II CR program on 30-, 60-, and 180-day readmission and short-term observation (STO) rates. Based on previous studies, it was hypothesized that participants in CR would have lower readmission and STO rates at 30,60, and 180 days than non-participants. Additionally, it was hypothesized that men would have higher readmission and STO rates than women. 


\section{Methods}

Participants. The Institution Review Boards from Akron General Medical Center and Kent State University approved the study procedures. No participant consent was required because of the retrospective study format. A total of 90 subjects: 45 Phase II CR participants, and 45 non-participants, were retrospectively reviewed.

Experimental procedures. Hospital discharge information was collected during the months of January 2012-June 2013 for diagnoses of unstable angina, percutaneous coronary angioplasty (PCI), PCI/stent, myocardial infarction (MI), coronary artery bypass graft surgery (CABG), valve surgery, or heart failure (HF). All patients during this discharge period were referred to CR unless medically contraindicated, and all patients had the option to enroll in CR following their index hospitalization. Subjects who participated in CR all completed 36 outpatient Phase II therapy sessions. As part of the CR therapy, individuals exercised wearing a ScottCare VersaCare 5-lead telemetry system (ScottCare Corp., 2014), and had the option to attend 14 education classes focused on risk factor modification and secondary disease prevention. Retrospectively, individuals who participated in CR were placed in the treatment group, and nonparticipants were placed in the control group.

The subjects' index admission and secondary diagnosis (if applicable) were noted, and through retrospective analysis of medical records utilizing MidasPlus, Inc. (Milliman, Inc., copyright 1984-2013), a database that records hospital discharges based on diagnosis-related group (DRG), and ChartMaxx (version 5.60.53, Medplus, Inc. copyright 2008), an electronic medical record (EMR) that records hospital utilization 
(i.e., hospitalizations, outpatient tests and procedures, and ED visits), hospital readmissions and STO were documented. Hospitalizations were defined in the hospital EMR as "INPT" and STO were coded as "STO."

\section{Data Analysis}

All statistical analyses were performed using SPSS $^{\complement}$ Version 22.0. Demographic data for all subjects are shown in Table 1 (in Chapter 3 ), and are shown as mean \pm SD. A Mann-Whitney U non-parametric test was utilized, with participant status as the grouping variable, to determine if differences existed in 30-, 60-, and 180-day readmission and STO rates between CR participants and non-participants. A 2-tailed independent $t$-test was used to determine if the number of lesions treated differed in both groups. An independent samples $t$-test was performed to ascertain if the total number of hospital readmissions was affected by participating in CR. Statistical significance was set with an alpha level of $p=$ 0.05. Relative risk ratios by participant status were calculated for readmissions and STO at 30,60 , and 180 days.

\section{Results}

A total of 90 subjects (participants $n=45$; non-participants $n=45$ ) were retrospectively reviewed in this study. The average age of the entire cohort was $64.7 \pm$ 9.9 years, with a total of 62 males and 28 females being reviewed in the analysis.

Readmission rates. Figure 1 depicts readmission rates for CR participants and non-participants at 30,60, and 180 days. Thirty-day readmission rates were higher for CR non-participants than participants (20\% vs. 6.7\%), which approached statistical significance $(\mathrm{U}=877.500, p=.064)$. Sixty-day readmission rates were higher for CR 


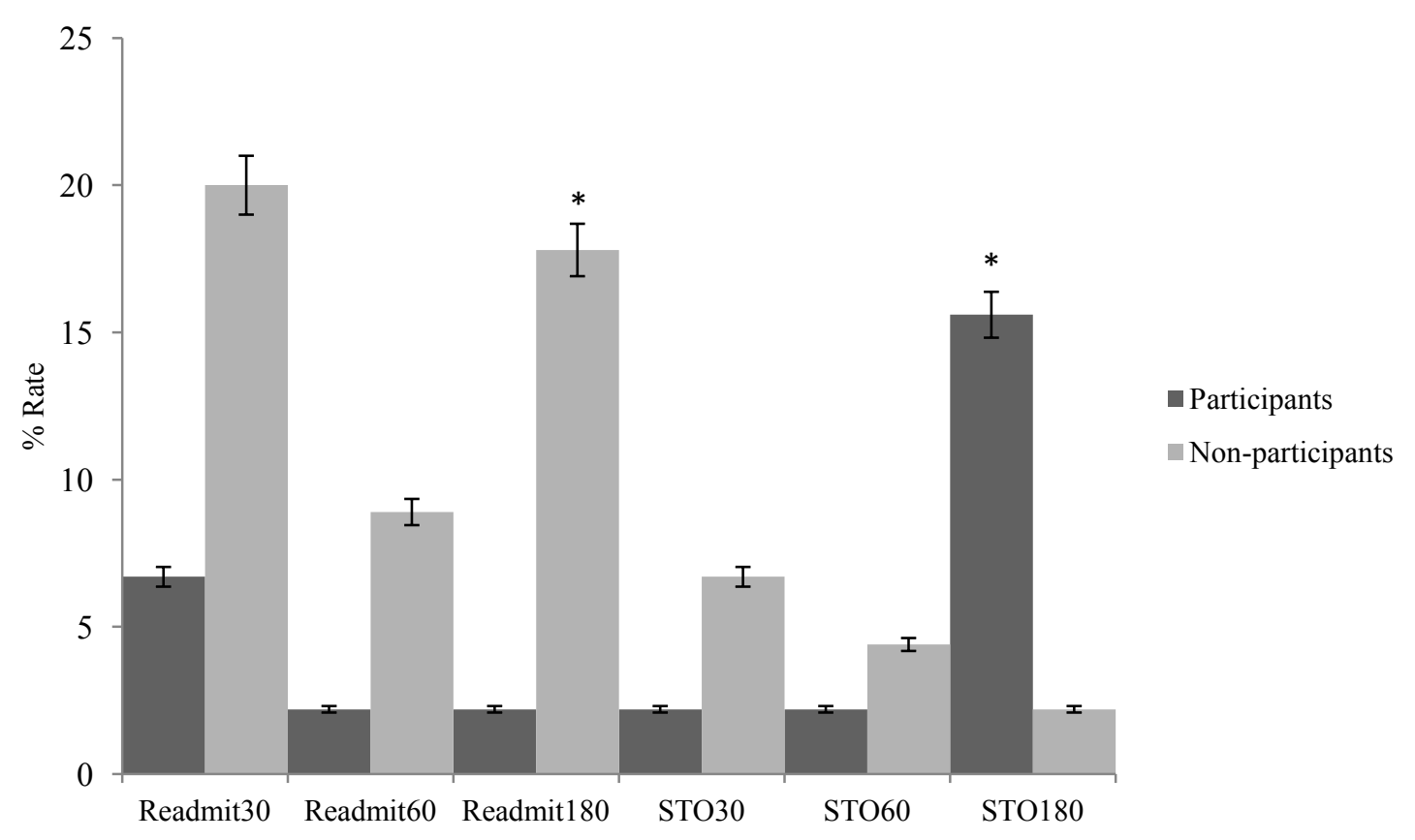

Figure 1. Readmission and STO rates for participants and non-participants at 30,60, and 180 days. *indicates statistical significance at $p<.05$.

non-participants than participants ( $8.9 \%$ vs. $2.2 \%)$ but were not statistically significant (U $=945, p=.170)$. One hundred eighty-day readmission rates were significantly higher for CR non-participants than participants $(17.8 \%$ vs. $2.2 \% ; \mathrm{U}=855, p=.014)$.

STO rates. Figure 1 depicts STO rates for CR participants and non-participants at 30,60, and 180 days. Thirty-day STO rates for CR non-participants were higher than participants $(6.7 \%$ vs. $2.2 \%)$ but were not statistically significant $(\mathrm{U}=967.500, p=.309)$. STO rates at 60 days were slightly higher in CR non-participants than participants $(4.4 \%$ vs. $2.2 \%)$ but were not statistically significant $(U=990, p=.559)$. One hundred eighty-day STO rates were significantly higher for CR participants than non-participants $(15.6 \%$ vs. $2.2 \% ; \mathrm{U}=877.500, p=.027)$. 
Number of treated lesions. Participants in CR had more than twice the number of atherosclerotic lesions treated than non-participants (2.1778 vs. 1.0889), which was statistically significant $[\mathrm{F}(88)=11.047, p=.001]$.

Total number of readmissions. Non-participants in CR had 5 times as many readmissions (.5556 vs. .1111) as those who participated in CR, which was statistically significant $[\mathrm{F}(88)=18.781, p<.001]$.

Relative risk for readmission. Table 2 demonstrates the relative risk for readmission and STO at 30, 60, and 180 days. Non-participants were 1.6 times more likely to readmit at 30 and 60 days than participants, although neither was statistically significant $\left[\left(\mathrm{x}^{2}(\mathrm{df}=1)=3.462, p=.063 ; \mathrm{x}^{2}(\mathrm{df}=1)=1.906, p=.167\right]\right.$. Non-participants were nearly twice as likely to readmit at 180 days than participants, which was statistically significant $\left[\mathrm{x}^{2}(\mathrm{df}=1)=6.049, p=.014\right]$. Non-participants were more likely at 30 and 60 days for STO $(\mathrm{RR}=1.536$ and 1.349 , respectively) than non-participants, although neither finding was statistically significant $\left[\mathrm{x}^{2}(\mathrm{df}=1)=\right.$ $\left.1.047, p=.306 ; \mathrm{x}^{2}(\mathrm{df}=1)=.345, p=.557\right]$. Participants were nearly twice as likely at 180 days for $\mathrm{STO}(\mathrm{RR}=1.888)$ than non-participants, which was statistically significant $\left[\mathrm{x}^{2}(\mathrm{df}=1)=4.939, p=.026\right]$.

Readmission and STO rates by gender. There were no differences in readmission rates at 30,60 , or 180 days $(p=.625, p=.660, p=.880$, respectively) or STO rates at 30,60 , or 180 days $(p=.788, p=.933, p=.697$, respectively) by gender. 
Table 2

Relative Risk Estimate By Participation Status and Timeframe

\begin{tabular}{llccc}
\hline \multicolumn{1}{c}{ Condition } & Timeframe & Risk Estimate & $95 \%$ CI Upper & 95\% CI Lower \\
\hline Participant & Readmit30 & .464 & .171 & 1.264 \\
Non-participant & Readmit30 & 1.625 & 1.084 & 2.437 \\
Participant & Readmit60 & .386 & .066 & 2.257 \\
Non-participant & Readmit60 & 1.659 & 1.016 & 2.709 \\
Participant & Readmit180 & $.205^{*}$ & .032 & 1.312 \\
Non-Participant & Readmit180 & $1.946^{*}$ & 1.397 & 2.710 \\
Participant & STO30 & .489 & .088 & 2.701 \\
Non-participant & STO30 & 1.536 & .838 & 2.814 \\
Participant & STO60 & .659 & .131 & 3.310 \\
Non-participant & STO60 & 1.349 & .589 & 3.087 \\
Participant & STO180 & $1.888^{*}$ & 1.330 & 2.681 \\
Non-participant & STO180 & $.233^{*}$ & .037 & 1.473 \\
\hline
\end{tabular}

Note. Relative risk estimates based on condition (participant/non-participant) and timeframe (30,60, 180 day readmission and $\mathrm{STO}) . *$ indicates statistical significance at $p<.05$.

\section{Discussion}

The purpose of this study was to investigate the effects of participating in cardiac rehabilitation on 30,60, and 180-day readmission rates. We hypothesized that 30,60, and 180-day readmission rates would be lower for individuals participating in Phase II CR than non-participants. We also hypothesized that readmission rates would be higher 
in men than women, and that individuals who were privately insured would have lower readmission rates than publicly insured individuals. The findings of this study were: (a) Participants in CR had significantly lower readmission rates at 180 days than non-participants, but higher 180 STO rates than non-participants; (b) Participants in CR had significantly more atherosclerotic lesions treated (with the index hospitalization) than non-participants; (c) Non-participants were at a significantly higher risk for readmission at 180 days than participants in $\mathrm{CR}$, and participants were at a significantly higher risk for STO at 180 days than non-participants; and (d) There was no difference in readmission or STO rates for men and women at any time point.

Readmission rates and participation in cardiac rehabilitation. Prior research by Canyon and Meshgin (2008) suggested reductions in admission rates at 12 months post-index hospitalization for individuals participating in CR. Our data suggest that the benefits of CR may be realized at a much earlier timeframe (180 days). We did not observe a significant difference in 30-day readmission rates by condition. This is most likely a result of the amount of time required to enroll patients into CR. Most patients who receive a non-surgical intervention (i.e., percutaneous intervention with stent) may begin CR 2-3 weeks post-procedure; however, surgical patients (i.e., coronary artery bypass graft surgery) often begin CR 4-8 weeks post-surgery. It is likely then, that the benefits of CR would not be realized during the first 30-60 days post-hospitalization.

Participants had 5 times fewer admissions during the 180 days post-index hospitalization than non-participants. Canyon and Meshgin (2008) noted that CR participants had fewer readmissions at 12 months and shorter length of stay (LOS) with 
subsequent hospitalizations than non-participants. With respect to the total number of readmissions, our findings are consistent with the findings from Canyon and Meshgin (2008).

Number of significant lesions in CR participants. CR participants in this study had twice as many high-grade lesions intervened on than non-participants. This is of particular interest because it indicates that the participants had a higher severity of CAD than non-participants. The potential severity of CAD may serve as motivator or predictor for CR participation, an area warranting further study.

Prior research suggests that individuals presenting with an ST elevation myocardial infarction (STEMI) or had reperfusion therapy had higher enrollment rates than those who did not present with STEMI or require reperfusion therapy (Dunlay et al., 2009). Despite having more atherosclerotic lesions intervened on and potentially more severe CAD, CR participants had lower readmission rates at each measured time frame. This may be explained by automatic order implementation of inpatient Phase I CR, which prior research has shown significantly increases the likelihood for enrollment and participation in outpatient Phase II CR (Dunlay et al., 2009; Higgins et al., 2008).

Risk for readmission and STO. The current research demonstrated lower readmission rates at 30,60 , and 180 days for CR participants than non-participants. Additionally, CR participation reduced readmission risk across all time points by $53 \%$, $61 \%$, and $79.6 \%$ at 30,60 , and 180 days post-hospitalization, with statistical significance at 180 days. Non-participants were $62.5 \%, 65.9 \%$, and $94.6 \%$ more likely to be readmitted at 30,60, and 180 days, respectively, with statistical significance at 180 days. 
The present research suggests that $\mathrm{CR}$ may reduce readmission risk in the short-term (i.e., 30-60 days); however, significant readmission risk reduction occurs at 180 days post-discharge. Participating in CR improves CV strength and endurance, which helps to decrease mortality and morbidity. Additionally, patients attend education classes focusing on secondary prevention, so that they are actively engaging in CV risk factor reduction.

Conversely, CR participants had nearly twice as many STO encounters as non-participants. Participating in CR reduced STO risk by $50.1 \%$ and $34.1 \%$ at 30 and 60 days, and non-participants increased the risk for STO encounters by $53.6 \%$ and $34.9 \%$ during the same timeframes. At 180 days, CR participants were $88.8 \%$ more likely to have an STO encounter than non-participants and non-participants were $76.7 \%$ less likely to have an STO encounter than CR participants. There are several plausible explanations for this. First, CR participants are less likely to readmit at all timeframes than non-participants. This is despite having twice as many significant lesions intervened on than non-participants. By participating in $\mathrm{CR}$, it is possible that the need for hospitalization was reduced to the point where a medical complication requiring medical attention merely needed observation (lasting 48 hours or less), rather than a full hospitalization. Second, the risk for STO in the non-participant cohort may be reflective of a higher rehospitalization rate in this group. Fewer non-participants have STO encounters at 180 days and reduced risk because those who would have been at risk for STO encounters may have had prior readmissions to address medical complications. 
Therefore, it is conceivable that, at 180 days, these individuals were finally stabilized medically.

Readmission rates by gender. We hypothesized that readmission and STO rates would be higher in men than women. There were no differences in readmission or STO rates at 30,60, or 180 days. Prior research by Jencks et al. (2009) showed men were more likely to readmit than women, whereas Stewart et al. (2000) found women were more likely to be readmitted post-CABG surgery. Our findings suggest that there is a standardization of care, especially with the usage of core measure medications (i.e., aspirin, anti-platelet, beta-blocker, angiotensin converting enzyme inhibitor, and statin), along with improvements in surgical and non-surgical interventions may contribute to our findings.

The present study has several limitations. First, this was a study of a single medical center. The findings from this study are specific to the medical center where data collection took place. As such, further research should investigate multiple medical centers, to determine if the findings from this study are consistent in a larger, multi-center study. Second, it was assumed that participants in this study utilized the medical center where the study was conducted for all medical care, including hospital admissions and STO encounters. Because of privacy laws, we were unable to access medical records from other institutions without obtaining consents and waivers from all participants. If a retrospective, multi-center trial investigating similar variables were studied, consents would be required from all participants. Additionally, other variables such as factors 
affecting motivation and compliance should be investigated to determine if CR utilization could be improved.

In conclusion, participating in CR significantly reduces the risk for, and has lower readmission rates than deferring $\mathrm{CR}$ participation, at 180 days post-index hospitalization. Participants in CR had significantly higher risk for, and incidence of, STO observation than non-participants at 180 days post-hospitalization. Despite the higher STO observation rates, this may indicate the need for further monitoring without requiring hospitalization. Men and women have similar readmission and STO rates across 30, 60, and 180 days, possibly indicating that gender does not affect care (i.e., standardization or uniformity in care). 


\title{
CHAPTER V
}

\section{$2^{\text {nd }}$ MANUSCRIPT}

\section{Predictors for Participation, Readmission and Short-Term Observation at 30, 60, and 180 Days Post-Hospitalization in Cardiac Rehabilitation Participants and Non-Participants}

\begin{abstract}
Cardiac rehabilitation (CR) is a vastly underutilized service following invasive and non-invasive interventions in the treatment of coronary artery disease. Despite the many benefits of CR, approximately half of those eligible are referred, and fewer than $20 \%$ actually enroll. The purpose of this study was to determine factors for participation in $\mathrm{CR}$ as well as predictors for hospital readmission and short-term observation (STO) at 30, 60, and 180 days post-index hospitalization, and to determine readmission and STO rates differed by insurance status (i.e., type, insured status). Two hundred twenty subjects (participants $n=45$; non-participants $n=175$ ) retrospectively participated in the study. All subjects were referred to Phase II cardiac rehabilitation (CR) following a qualifying cardiac diagnosis, and given the option to participate. Thirty-day readmission predictors included acute heart failure (HF) with admission, no prescription for aspirin at discharge, and no myocardial infarction (MI) with admission. Sixty-day readmission predictors for readmission at 60 days were readmission at 30 days, age, number of lesions treated, history of depression, unemployed status, and no beta-blocker prescription at discharge. One hundred eighty-day readmission predictors of readmission were length of stay (LOS), history of COPD, history of depression, prescribed antiplatelet at discharge,
\end{abstract}


being uninsured, number of atherosclerotic lesions at time of hospitalization, non-hypertensive, and no prescription for angiotensin converting enzyme (ACE) inhibitor or angiotensin receptor blocker (ARB) at discharge. There were no significant predictors for 30-day STO. Predictors for 60-day STO were index LOS, tobacco use, and no acute HF with index admission. Predictors for 180-day STO were cardiac arrest upon arrival, chest pain with admission, no $\mathrm{ACE} / \mathrm{ARB}$ at discharge, type of $\mathrm{MI}, \mathrm{CR}$ participation, tobacco use, and insurance status. The strongest predictors for participating in CR were marital status, being non-hypertensive, non-smoker, and number of treated lesions.

\section{Introduction}

Cardiac rehabilitation (CR) is considered an effective secondary prevention program in the management of coronary artery disease (CAD). There are many benefits to participating in a monitored CR program. Artham et al. (2008) noted a 35\% increase in metabolic equivalent (MET) levels, a 15\% increase in peak oxygen consumption $\left(\mathrm{VO}_{2 \text { peak }}\right)$, and an $11 \%$ increase in anaerobic threshold.

Despite the many benefits of CR, it is a vastly underutilized service. According to the American Heart Association Get With the Guidelines (AHA GWTG 2009), only 56\% of all eligible patients were referred to CR. Fifty three percent of patients with AMI were referred, $58 \%$ of patients with percutaneous coronary intervention (PCI) were referred, and $74 \%$ of patients with coronary artery bypass graft (CABG) surgery were referred. Even worse is the attendance rate in CR programs. There is an $18.7 \%$ attendance rate in CR programs in the United States (Lavie et al., 2009). Worldwide, only $15-30 \%$ of eligible patients attend CR (Neubeck et al., 2011). 
Many factors can affect CR participation, namely patient-centered factors (i.e., health insurance status), health-related factors (i.e., multiple comorbidities), and institutional factors (no CR program available). Dunlay et al. (2009) identified patient characteristics that increased the likelihood of CR referral and enrollment, such as age, gender, diagnosis, if the physician practiced at the hospital where the patient was admitted, as well as other variables.

Healthcare has placed an increased emphasis on providing quality care. One of the quality indicators being watched very closely by hospitals and the Centers for Medicare and Medicaid Services (CMS) is 30-day readmission rates. Hospitals are subjected to financial penalties in the form of reduced reimbursements if 30 -day readmission rates for specific diseases: acute myocardial infarction (AMI), pneumonia $(\mathrm{PN})$, congestive heart failure (CHF), are above an established benchmark. Prior research estimated that up to $76 \%$ of 30 -day readmissions are preventable (American Hospital Association, 2011; MedPAC, 2007; Jencks et al., 2009).

Prior studies have investigated predictors for 30-day readmissions based on diagnosis (i.e., MI, percutaneous intervention) as well as predictors for participation in CR. Past research has focused on predictors for 30-day readmission, but have not addressed the issue of short-term observation (STO), which is medical observation in a hospital lasting 48 hours or less. The purpose of this study is to identify predictors for readmission and short-term observation at 30,60, and 180 days, to determine predictors for participation, and to investigate if insurance status influences readmission or STO rates. Based on previous research, it was hypothesized that age, number of lesions 
treated, gender, and insurance status would be predictors for participation in CR. Additionally, it was hypothesized that a history of chronic obstructive disease (COPD), diagnosis, new onset of HF, and insurance status would be predictors for 30-, 60-, and 180-day readmissions and STO. Lastly, it was hypothesized that privately insured individuals would have significantly lower readmission and STO rates at 30,60, and 180 days, than individuals with public or no insurance at all.

\section{Methods}

Participants. The Institution Review Boards from Akron General Medical Center and Kent State University approved the study procedures. No participant consent was required because of the retrospective study format. A total of 221 subjects, 45 Phase II CR participants, and 176 non-participants were retrospectively reviewed.

Experimental procedures. Hospital discharge information was collected during the months of January 2012-June 2013 for diagnoses of unstable angina, percutaneous coronary angioplasty (PCI), PCI/stent, myocardial infarction (MI), coronary artery bypass graft surgery (CABG), valve surgery or heart failure (HF) using an electronic medical record (ChartMaxx, version 5.60.53, Medplus, Inc., copyright 2008. All patients during this discharge period were referred to CR unless medically contraindicated, and all patients had the option to enroll in CR following their index hospitalization. Subjects who participated in CR all completed 36 outpatient Phase II therapy sessions. As part of the CR therapy, individuals exercised wearing a ScottCare VersaCare 5-lead telemetry system (ScottCare Corp., 2014), and had the option to attend 14 education classes focused on risk factor modification and secondary disease prevention. Retrospectively, 
individuals who participated in CR were placed in the treatment group, and non-participants were placed in the control group.

The subjects' index admission and secondary diagnosis (if applicable) were noted, and through retrospective analysis of medical records utilizing MidasPlus, Inc.

(Milliman, Inc., Copyright 1984-2013), a database that records hospital discharges based on diagnosis-related group (DRG), and ChartMaxx (version 5.60.53, Medplus, Inc. Copyright 2008) an electronic medical record (EMR) that records hospital utilization (i.e., hospitalizations, outpatient tests and procedures, and ED visits), hospital readmissions and STO were documented. Hospitalizations were defined in the hospital EMR as "INPT" and STO were coded as "STO."

\section{Data Analysis}

All statistical analyses were performed using SPSS $^{\complement}$ Version 22.0. Demographic data for all subjects is shown in Table 3, and is shown as mean + SD. Stepwise regression was utilized to determine which variables predicted readmission and STO at 30, 60, and 180 days post-index hospitalization, which included: primary diagnosis, secondary diagnosis, 30-day readmission or STO (as a predictor for 60- or 180-day readmission or STO), 60-day readmission or STO (as a predictor for readmission or STO at 180 days), new onset of HF (with index admission), receiving aspirin at discharge, having an MI with index admission, chest pain at admission, cardiac arrest at arrival (of index admission), number of significant lesions treated (at index admission), transfer from another facility, CR participation status, type of MI (if applicable), age, age category, gender, ethnicity, marital status, employment status, index length of stay, type of 
Table 3

Demographic Data for Entire Cohort of Participants and Non-Participants

\begin{tabular}{|c|c|}
\hline & Value \\
\hline Men: \# participants & 36 \\
\hline Men: \# non-participants & 114 \\
\hline Age & $65.8 \pm 11.17$ \\
\hline Women: \# participants & 9 \\
\hline Women: \# non-participants & 62 \\
\hline Age: participants & $65.8 \pm 11.17$ \\
\hline Age: non-participants & $63 \pm 12.59$ \\
\hline \# Private insurance & $125(56.3 \%)$ \\
\hline \# Public insurance & $35(15.8 \%)$ \\
\hline \# Public/private secondary insurance & $35(15.8 \%)$ \\
\hline \# Uninsured & $26(11.7 \%)$ \\
\hline \# Tobacco use & $81(36.5 \%)$ \\
\hline \# Hypertensive & $174(78.4 \%)$ \\
\hline \# Diabetic & $78(35.1 \%)$ \\
\hline \# Dyslipidemia & $217(97.7 \%)$ \\
\hline$\# \mathrm{BMI}<18$ & $3(1.4 \%)$ \\
\hline \# BMI 18-24.9 & $41(18.5 \%)$ \\
\hline \# BMI 25-29.9 & $77(34.7 \%)$ \\
\hline$\# \mathrm{BMI}>30$ & $100(45 \%)$ \\
\hline \# Sedentary & $127(57.2 \%)$ \\
\hline \# History of depression & $52(23.4 \%)$ \\
\hline \# Previous CAD event & $90(40.5 \%)$ \\
\hline
\end{tabular}

Note. Expressed as mean \pm SD. 
insurance, tobacco use, hypertension, diabetes, dyslipidemia, BMI, BMI category, sedentary lifestyle, history of depression, previous cardiac event, orthopedic issues, receiving antiplatelet at discharge, receiving beta blocker at discharge, receiving ACE inhibitor or ARB at discharge, receiving lipid agent at discharge, and history of COPD. Stepwise regression was also used to calculate predictors for participating in CR. A non-parametric Mann-Whitney U was performed to establish if readmission and STO rates at 30, 60, and 180 days were affected by insurance status. Fisher's LSD post-hoc test was then utilized to determine under which insurance conditions the differences occurred. Statistical significance was set an alpha level of $p=0.05$.

\section{Results}

Predictors for Readmission and STO. Stepwise regression was utilized to determine predictors for readmission and short-term observation. Each of the models presented are statistically significant with the exception of predictors for 30-day STO, and are listed in Table 4. The strongest predictors for 30-day readmission were a new onset of HF, no aspirin prescribed at discharge, and no diagnosis of MI during the index hospitalization, accounting for approximately $13 \%$ of the variance for 30 -day readmissions. New onset of HF was the strongest predictor, accounting for $8.3 \%$ of the variability. The most powerful predictors for 60-day readmission were readmission at 30 days, no beta-blocker prescribed at discharge, being unemployed, increasing age, an increased number of treated lesions, and a history of depression, accounting for $20.5 \%$ of the variability. Thirty-day readmissions were the strongest predictor, accounting for $7.4 \%$ of the variability. The strongest predictors for 180 -day readmission were decreased 
Table 4

Predictors for Readmission and STO at 30, 60, and 180 Days

\begin{tabular}{|c|c|c|c|c|c|c|}
\hline Factor & $\begin{array}{c}\text { Mod } \\
\text { el }\end{array}$ & Variable & Adjusted R square & $\mathrm{df}$ & $\mathrm{F}$ & Significance \\
\hline \multirow{3}{*}{$\begin{array}{l}\text { 30-Day } \\
\text { Readmission }\end{array}$} & 1 & New Onset of HF & .083 & 219 & 20.793 & .000 \\
\hline & 2 & Aspirin & .107 & 219 & 14.080 & .000 \\
\hline & 3 & MI & .131 & 219 & 10.825 & .000 \\
\hline \multirow{6}{*}{$\begin{array}{l}\text { 60-Day } \\
\text { Readmission }\end{array}$} & 1 & Readmit at 30 days & .070 & 219 & 17.441 & .000 \\
\hline & 2 & Beta blocker & .106 & 219 & 14.043 & .000 \\
\hline & 3 & Employed & .129 & 219 & 11.803 & .000 \\
\hline & 4 & Age & .151 & 219 & 10.733 & .000 \\
\hline & 5 & Treated lesions & .170 & 219 & 9.955 & .000 \\
\hline & 6 & Depression history & .182 & 219 & 9.147 & .000 \\
\hline \multirow{8}{*}{$\begin{array}{l}\text { 180-Day } \\
\text { Readmission }\end{array}$} & 1 & LOS index & .083 & 219 & 20.762 & .000 \\
\hline & 2 & COPD history & .145 & 219 & 19.627 & .000 \\
\hline & 3 & Depression history & .173 & 219 & 16.299 & .000 \\
\hline & 4 & Significant lesions & .195 & 219 & 14.290 & .000 \\
\hline & 5 & Antiplatelet & .209 & 219 & 12.586 & .000 \\
\hline & 6 & Insurance & .221 & 219 & 11.359 & .000 \\
\hline & 7 & $\mathrm{ACE}$ or $\mathrm{ARB}$ & .233 & 219 & 10.512 & .000 \\
\hline & 8 & HTN & .245 & 219 & 9.888 & .000 \\
\hline \multirow[t]{3}{*}{ 60-Day STO } & 1 & LOS index & .027 & 219 & 7.174 & .008 \\
\hline & 2 & Tobacco use & .044 & 219 & 6.015 & .003 \\
\hline & 3 & New onset HF & .056 & 219 & 5.369 & .001 \\
\hline \multirow[t]{8}{*}{ 180-Day STO } & 1 & $\begin{array}{l}\text { Cardiac arrest at } \\
\text { arrival }\end{array}$ & .036 & 219 & 9.120 & .003 \\
\hline & 2 & $\begin{array}{l}\text { Chest pain on } \\
\text { admission }\end{array}$ & .063 & 219 & 8.406 & .000 \\
\hline & 3 & $\mathrm{ACE}$ or ARB & .086 & 219 & 7.830 & .000 \\
\hline & 4 & MI type & .101 & 219 & 7.147 & .000 \\
\hline & 5 & Tobacco use & .119 & 219 & 6.906 & .000 \\
\hline & 6 & $\begin{array}{l}\text { Rehab } \\
\text { participation }\end{array}$ & .142 & 219 & 7.021 & .000 \\
\hline & 7 & New onset HF & .162 & 219 & 7.057 & .000 \\
\hline & 8 & Insurance & .174 & 219 & 6.779 & .000 \\
\hline
\end{tabular}

Note. Significant predictors for readmission and STO at 30, 60, and 180 days, utilizing stepwise regression $(p<.05)$. No significant predictors for STO at 30 days were found. 
index LOS, history of COPD, history of depression, decreased number of significant lesions $(\geq 75 \%)$, prescribed antiplatelet therapy at discharge, uninsured status, and no prescription for ACE or ARB therapy, accounting for $27.3 \%$ of the variability. A decrease in LOS of .024 from mean LOS was the chief predictor, followed closely by history of COPD, accounting for $8.7 \%$ and $6.6 \%$, respectively. Stepwise regression showed no significant predictors for 30-day STO. The principal predictors for 60-day STO were a decreased index LOS, tobacco use, and no new onset of HF during the index hospitalization, accounting for $6.9 \%$ of the variability. A decrease in LOS of .009 from mean LOS was the strongest predictor, accounting for $3.2 \%$ of the variability. The leading predictors for 180-day STO were cardiac arrest at arrival, chest pain with admission, no ACE or ARB prescribed at discharge, having a non-ST elevation $\mathrm{MI}$ (NSTEMI), tobacco use, participation in CR, new onset of HF, and being uninsured, accounting for $20.4 \%$ of the variability. Cardiac arrest at arrival was the key predictor, accounting for $4 \%$ of the variability $\left(Y_{\text {predicted }}=b 0+b 1 * x 1+b 2 * x 2+b 3 * x 3+b 4 * x 4\right)$.

Predictors for CR participation. Table 5 lists the significant predictors for CR participation. Four variables were significant predictors for CR participation: decreased number of treated lesions, no tobacco use, being married, and being non-hypertensive, accounting for $17.4 \%$ of the variability. A decrease in the number of treated lesions from the mean accounted for $6.5 \%$ of the variability and was the largest predictor for participation in $\mathrm{CR}$, suggesting that individuals with fewer treated atherosclerotic lesions were more likely to participate in CR. 
Table 5

Predictors for Participation in Cardiac Rehabilitation

\begin{tabular}{clcccc}
\hline Model & Variable & Adjusted R square & df & F & Significance \\
\hline 1 & Treated lesions & .061 & 219 & 15.191 & .000 \\
2 & Tobacco use & .100 & 219 & 13.227 & .000 \\
3 & Marital status & .129 & 219 & 11.839 & .000 \\
4 & HTN & .158 & 219 & 11.300 & .000 \\
\hline
\end{tabular}

Note. Significant predictors for participation in cardiac rehabilitation $(p<.05)$

Readmission and STO by insurance status. A non-parametric Mann-Whitney U showed no main effect differences in readmission rates at 30, 60, and 180 days by insurance $(p=.270, p=.209$, and $p=.054$, respectively). There was a significant main effect for STO rates by insurance for 60 day STO and 180 day STO, [F (3) = 3.685, $p<$ .001 , and F $(3)=2.719, p=.049$, respectively]. Post-hoc analysis revealed public insurance had significantly lower 60 -day STO rates than private insurance $(p=.002)$ and no insurance $(p=.041)$. At 180 days, public with secondary insurance had significantly lower STO rates than private insurance, public insurance and no insurance $(p=.016, p=$ .018 , and $p=.05$, respectively).

\section{Discussion}

The purpose of this study was to determine predictors for readmission and STO at 30, 60, and 180 days post-index hospitalization. We hypothesized that age, number of lesions treated, gender, and insurance status would be significant predictors for CR 
participation. We also hypothesized that COPD history, new onset of HF, and insurance status would be significant predictors for readmission and STO, and that private insurance would have significantly lower readmission and STO rates than public insurance. The findings of this study were: (a) The strongest single predictors for readmission at 30,60 , and 180 , days were new onset of $\mathrm{HF}$, readmission at 30 days, and decreased LOS from mean, respectively. There was no statistically significant predictor for 30-day STO. The strongest single predictors for STO at 60, and 180 days were decreased LOS from mean, and cardiac arrest at arrival, respectively; (b) The 4 significant predictors for CR participation are decreased lesions treated from mean, smoke-free status, being married, and non-hypertensive status; and (c) Public insurance had lower 60-day readmission rates than public with secondary insurance, and public with secondary insurance had lower 180 -day readmission rates than private insurance. Public insurance had significantly lower 60 STO rates than private insurance, and public with private secondary insurance had significantly lower 180 STO rates than private, public, and no insurance.

Predictors for readmission and STO. Prior research investigated predictors for 30-day readmission, either by diagnosis or by intervention (J. Brown et al., 2013; Jencks et al., 2009; Jiang \& Wier, 2010; Kociol, 2011; Kociol et al., 2012; Ricciardi et al., 2012; Stewart et al., 2000). Our findings were consistent with the findings from these prior studies, although there was no single, universal predictor for readmission. The strongest predictors for readmission and STO appear to be a combination of patient and 
institutional variables, which are dynamic, change over time, and represent a relatively small percentage of the contribution towards readmission and STO.

Conversely, some of our findings contradict prior research. Kaboli et al. (2012) observed that reduced LOS did not correlate with 30-day readmission rates. In fact, LOS was reduced for several disease states while simultaneously reducing 30-day readmission rates and overall mortality. We found that reduced LOS below mean was the most significant predictor for both readmission at 180 days and STO at 60 days. There are many possible explanations for this, including patients not being medically ready for discharge, multiple comorbidities, and access to post-hospitalization care. Ricciardi et al. (2012) found that women were more likely to readmit than men; in this study, gender was not a significant predictor for readmission or STO. This may be due to improvements in medical care delivery, standardization of care, and caregivers being more cognizant of cardiac signs and symptoms in both men and women.

Predictors for CR participation. CR participation was predicted by the absence of 2 cardiac risk factors (tobacco use and hypertension), a medical variable (number of lesions treated), and a psychosocial variable. Prior research by Dunlay et al. (2009) identified, among other variables, gender and age as significant predictors, variables that were significant predictors in the current study. We observed that a decreased number of treated lesions below mean is a predictor for CR participation, and suggests that individuals with less severe $\mathrm{CAD}$ are participating in $\mathrm{CR}$, when it could be argued that those with the most severe CAD stand to gain the most through participation. We also 
observed that these 4 variables contributed only $17 \%$ to predicting participation indicates that other variables affect CR enrollment.

Readmission and STO by insurance status. Insurance type was not a significant factor in 30-day readmissions. This is in stark contrast to findings from Jiang and Wier (2010) and Wier et al. (2011), who found significantly higher readmission rates with public insurance compared with private insurance. This was in part to patient demographics who hold public insurance. Publicly insured individuals tend to be older (i.e., retired), have multiple comorbidities, and have other socioeconomic factors that can increase the risk for readmission. Individuals with private insurance tend to be younger, employed, and may be in better general health than those with public insurance.

In addition to studying private versus public insurance, we also studied public insurance with private secondary insurance and non-insured individuals. Private insurance had a higher occurrence of STO at 60 and 180 days than public and public with private secondary, respectively. The higher episodes of readmission and STO with private insurance may reflect a shift towards a consumer-driven health plan (CHDP), where policyholders have high out of pocket deductibles that must be met before benefits take effect. With these high deductibles, individuals may defer elective medical care, including preventative care, which could increase the need for hospitalization or STO.

Public insurance did have higher incidence of STO at 180 days than public with private secondary insurance, but otherwise had similar episodes of readmission as private insurance. More common is the publicly insured with private secondary insurance. The secondary insurance typically will cover medical expenses not covered by a traditional 
Medicare (which normally covers $80 \%$ of a contracted amount for services), so it essentially is a "hybrid" insurance plan (part public and part private insurance). Many public with private secondary plans have medical expenses covered at, or near, $100 \%$. This may make individuals more likely to seek preventative medical care and indicates that a socioeconomic component to readmissions and STO exists.

Surprisingly, uninsured individuals are not more likely to readmit than their insured (fully or partially) counterparts. A large part of this is likely that uninsured individuals are deferring elective medical care (preventative or otherwise) due to financial considerations. We observed that individuals have higher 180-day STO rates than public with private secondary, which further validates this point. Even so, there may be an effort to treat uninsured individuals in an economic manner that is cost-effective.

This study had several limitations. The study occurred at a single medical center. As such, it was assumed that all individuals received inpatient and STO care at the hospital where the study was conducted. In order to circumvent this, a multi-center protocol should be considered, so that medical records could be obtained and analyzed from all medical centers participating in their care. Because of privacy laws, we were unable to access medical records from other institutions without obtaining consents and waivers from all participants. In addition, no variables addressing education or motivation were addressed. The current study identified some predictors for participation, but clearly more research needs to be done with respect to motivation, as there appear to be variables affecting CR participation that were not investigated. 
In conclusion, there are many predictors for readmission, which seem to be specific to the respective timeframe (i.e., new onset of $\mathrm{HF}$ is a predictor for 30 -day readmission, but not for 60-day readmission) and are widely varied. Although several variables were identified as predictors for CR participation, more research needs done in this area to better quantify those components that are contributory to CR enrollment. Insurance status is a significant predictor for readmission at 60 days, and the type of insurance (or none) likely has an effect on hospital and healthcare utilization. Further research should focus on healthcare utilization, especially elective and preventative care, as it pertains to insurance. 


\section{CHAPTER VI}

\section{SUMMARY}

To our knowledge, this is the first study that attempted to examine if participating in cardiac rehabilitation had any effect on readmission and short-term observation rates at 30, 60, and 180 days. The purpose of the present study was to determine if participating in an outpatient, Phase II cardiac rehabilitation program yielded lower readmission and short-term observation rates. A secondary goal was to investigate variables that predicted participation in cardiac rehabilitation, readmission, and short-term observation.

Lower readmission rates were observed in cardiac rehabilitation participants than non-participants at 30,60, and 180 days, with a statistically significant lower readmission rate at 180 days. It is worth noting that non-participants readmitted 5 times as often as participants. Additionally, participants had twice as many atherosclerotic lesions treated as non-participants, potentially indicating more severe coronary artery disease in the participant cohort. Cardiac rehabilitation participants had a statistically significant higher

rate of short-term observation than non-participants. It is worth noting that participants in non-participants readmitted short-term observations, lasting less than 48 hours in duration, are not considered a hospital admission.

Predictors for participation in cardiac rehabilitation included fewer atherosclerotic lesions below mean, smoke-free status, being non-hypertensive, and being married. There were multiple predictors for readmission and short-term observation, which were widely varied based on time (30,60, or 180 days) and condition (readmission or short-term observation). The strongest predictors accounted for a very small proportion 
of the variability, indicating that a multitude of factors, including some that were not investigated in the current research, can be predictive of readmission and short-term observation. Our study did not find a difference in readmission or short-term observation rates in men and women. There was a significant difference in the incidence of readmission and short-term observation by insurance status, at 30, 60, and 180 days. Contrary to previous research, private insurance had higher readmission episodes at 180 days and short-term observation at 60 days than other forms of insurance. Public with private insurance had higher readmission occurrences at 60 days, but lower readmission and short-term observation occurrences at 180 days than other insurance options. Uninsured status had higher episodes of short-term observation at 60 and 180 days than other types of insurance.

Cardiac rehabilitation participation was associated with lower readmission rates at 30, 60, and 180 days than non-participants. Many variables appear to influence hospital readmission and short-term observation. The previous literature focused on 30-day readmissions, but this was the first study that attempted to explore 30-, 60-, and 180-day readmission and short-term observations. As this research was conducted at a single medical center, future research should include a multi-center study to determine if the results from the current study can be generalized to a larger population, or if the results are unique to the study site. Furthermore, more research is needed to discern whether these variables are predictive of cardiac rehabilitation and readmissions. 


\section{REFERENCES}




\section{References}

Ades, P., Waldmann, M., McCann, W., \& Weaver, S. (1992). Predictors of cardiac rehabilitation participation in older coronary patients. Archives of Internal Medicine, 152, 1033-1035. doi: 10.1001/archinte.1992.00400170113021

Agency for Healthcare Research and Quality, Medical Expenditure Panel Survey. (2008). Healthcare quality report. Rockville, MD: Author. Retrieved from http://archive.ahrq.gov/research/findings/nhqrdr/nhqr08/methods/meps.html

Agency for Healthcare Research and Quality. (2013, November). National health care expenses in the U.S. civilian noninstitutionalized population, 2011. Statistical Brief \#425. Rockville, MD: Author.

Ali, A., Mehra, M., Lavie, C., Malik, F. S., Murgo, J. P., Lohmann, T. P. . . Milani, R. V. (1998). Modulatory impact of cardiac rehabilitation on hyperhomocysteinemia in patients with coronary artery disease and "normal" lipid levels. American Journal of Cardiology, 82, 1543-1545, A8.

American Cancer Society. (2015). Economic impact of cancer. Retrieved from http://www.cancer.org/Cancer/CancerBasics/economic-impact-of-cancer American College of Sports Medicine [ACSM]. (2010). ACSM's guidelines for exercise testing and prescription ( $8^{\text {th }}$ ed.). Philadelphia, PA: Lippincott Williams \& Wilkins.

American Heart Association. (2012). Heart disease and stroke statistics -2012 update: A report from The American Heart Association. Circulation, 125, e2-e220. doi: 10.1161/CIR.0b013e31823ac046 
American Heart Association. (2013). 2013 ACCF/AHA key data elements and definitions for measuring the clinical management and outcomes of patients with acute coronary syndromes and coronary artery disease. Journal of the American College of Cardiology, 61(9), 992-1025. doi: 10.1016/j.jacc.2012.10.005

American Hospital Association. (2011). Examining the drivers of readmissions and reducing unnecessary readmissions for better patient care. Washington, DC: TrendWatch.

Artham, S., Lavie, C., Milani, R., Chi, Y., \& Goldman, C. (2008). Benefits of exercise training in secondary prevention of coronary and peripheral arterial disease. Vascular Disease Prevention, 5, 156-168. doi:

http://dx.doi.org/10.2174/156727008785133818

Brown, J., Conley, S., \& Niles II, N. (2013). Predicting readmission or death after acute ST-elevation myocardial infarction. Clinical Cardiology, 1-6. doi: $10.1002 / \mathrm{clc} 22156$

Canyon, S., \& Meshgin, N. (2008). Cardiac rehabilitation reducing hospital readmissions through community based programs. Australian Family Physician, 37, 575-577.

Carney, R., Freedland, K., Miller, G., \& Jaffe, A. (2002). Depression as a risk factor for cardiac mortality and morbidity: A review of potential mechanisms. Journal of Psychosomatic Research, 53, 897-902.

Carney, R., Freedland, K., Sheline, Y., \& Weiss, E. (1997). Depression and coronary heart disease: A review for cardiologists. Clinical Cardiology, 20, 196-200. 
Centers for Disease Control. (2010). National vital statistics reports. Division of Vital Statistics, 60(3).

Centers for Medicare and Medicaid Services. (2011). Medicare Hospital Quality Chartbook 2011 performance report on readmission measures for acute myocardial infarction, heart failure, and pneumonia. Prepared by Yale New Haven Health System Corporation Center for Outcomes Research and Evaluation: Author.

Centers for Medicare and Medicaid Services. (2011). National Healthcare Expenditure Projections, 2011-2021. Office of the Actuary: Author.

Centers for Medicare and Medicaid Services. (2012). National Health Expenditures 2012-2022 Forecast Summary. Office of the Actuary: Author.

Centers for Medicare and Medicaid Services. (n.d.). Retrieved from https://www.cms.gov/Medicare/Medicare-Fee-for-ServicePayment/ACO/index.html?redirect=/ACO

Computer Sciences Corporation. (2012, July). Preventing hospital readmissions: The first test case for continuity of care (Issue Brief WA12_0155 HCG). Falls Church, VA: Author.

Corrections (2012). Circulation, 125, e1002. doi: 10.1161/CIR.0b013e31825e7519

Cortes, O., \& Arthur H. (2006). Determinants of referral to cardiac rehabilitation programs in patients with coronary artery disease: A systematic review. American Heart Journal, 151, 249-256. 
Cowie, C., Rust, K., Byrd-Holt, D., Eberhardt, M., Flegal, K., Engelgau, M. M. . . Gregg, E. W. (2006). Prevalence of diabetes and impaired fasting glucose in adults in the U.S. Population: National Health and Nutrition Examination Survey, 1999-2002. Diabetes Care, 29(6), 1263-1268. doi: 10.2337/dc06-0062

Curnier, D., Savage, P., \& Ades, P. (2005). Geographic distribution of cardiac rehabilitation programs in the United States. Journal of Cardiopulmonary Rehabilitation, 25, 80-84.

Dharmarajan, K., Hsieh, A., Lin, Z., Bueno, H., Ross, J., Horwitz, L. . . Krumholtz, H. M. (2013). Diagnoses and timing of 30-day readmissions after hospitalization for heart failure, acute myocardial infarction or pneumonia. Journal of the American Medical Association, 309(4), 355-363. doi: 10.1001/jama.2012.216476

Dimsdale, J. (2008). Psychological stress and cardiovascular disease. Journal of the American College of Cardiology, 51, 1237-1246. doi: 10.1016/j.jacc.2007.12.024.

Doe, V. (2009). Effect of cardiac rehabilitation in depressed versus nondepressed individuals on fitness, depression, and perceived exertion. Unpublished doctoral dissertation, Kent State University, Kent, $\mathrm{OH}$.

Dunlay, S., Witt, B., Allison, T., Hayes, S., Weston, S., Koepsell, E., \& Roger, V. L. (2009). Barriers to participation in cardiac rehabilitation. American Heart Journal, 158(5), 852-859. doi: 10.1016/j.ahj.2009.08.010

Eaton, D., Kann, L., Kinchen, S., Shanklin, S., Ross, J., Hawkins, J. . . Wechsler, H. (2010). Youth risk behavior surveillance-United States, 2009. Mortality and Morbidity Weekly Report Surveillance Summaries, 59(5), 1-142. 
Ervin, B. (2009). Prevalence of metabolic syndrome among adults 20 years of age and over, by sex, age, race and ethnicity, and body mass index: United States, 20032006. National Health Statistics Report, 13, 1-7.

Flegal, K., Carroll, M., Ogden, C., \& Curtin, L. (2010). Prevalence and trends in obesity among US adults, 1999-2008. Journal of the American Medical Association, 303, $235-241$.

Ford, E. S. (2011, June). Trends in the risk for coronary heart disease among adults with diagnosed diabetes in the U.S. Findings from the National Health and Nutrition Examination Survey, 1999-2008. Diabetes Care, 34(6), 1337-1343. doi: $10.2337 / \mathrm{dc} 10-2251$

Ford, E., Ajani, U., Croft, J., Critchley, J., Labarthe, D., Kottke, T. . . Capewell, S. (2007). Explaining the decrease in U.S. deaths from coronary disease, 1980-2000. New England Journal of Medicine, 356, 2388-2398.

Goodman, D., Fisher, E., Chang, C., Raymond, S., \& Bronner, K. (Eds.). (2013). The revolving door: A report of U.S. hospital readmissions. An analysis of Medicare data by the Dartmouth Atlas Project (Robert Wood Johnson Foundation, Section 1). Lebanon, NH: The Dartmouth Atlas Project.

Grace, S., Gravely-Witte, S., Brual, J., Monette, G., Suskin, N., Higginson, L. . . Stewart, D. E. (2008). Contribution of patient and physician factors to cardiac rehabilitation enrollment: a prospective multi-level study. European Journal of Cardiovascular Prevention and Rehabilitation 15, 548-556. 
Hambrecht, R., Wolf, A., Gielen, S., Linke, A., Hofer, J., Erbs, S. . . Schuler, G. (2000). Effect of exercise on coronary endothelial function in patients with coronary artery disease. New England Journal of Medicine, 342, 454-460.

Hamilton, G., \& Seidman, M. (1993). A comparison of the recovery period for women and after an acute myocardial infarction. Heart Lung, 22, 308-315.

Heart Disease and Stroke Statistics_-2012 Update: A Report From The American Heart Association (2011). Circulation, 125, e2-e220. doi:

10.1161/CIR.0b013e31823ac046

Heidenreich, P., Albert, N., Allen, L., Bluemke, D., Butler, J., Fonarow, G. . . Trogdon, J. C. (2013). Forecasting the Impact of heart failure in the United States: A policy statement from the American Heart Association. Circulation Heart Failure, 6, 606-619. doi: 10.1161/HHF.0b013e318291329a

Higgins, R., Murphy, B., Goble, A., Le Grande, M., Elliott, P., \& Worcester, M. (2008). Cardiac rehab program attendance after coronary artery bypass graft: Overcoming the barriers. Medical Journal of Australia, 188, 712-714.

Howard, G., Prineas, R., Moy, C., Cushman, M., Kellum, M., Temple, E. . . Howard, V. (2006). Racial and geographic differences in awareness, treatment and control of hypertension: The reasons for geographic and racial differences in stroke study. Stroke, 37(5), 1171-1178.

Jack, B., Chetty, V., Anthony, D., Greenwald, J., Sanchez, G., Johnson, A. . . Culpepper, L. (2009). A reengineered hospital discharge program to decrease 
rehospitalization: A randomized trial. Annals of Internal Medicine, 150(3), 178187.

Jencks, S., Williams, M., \& Coleman, E. (2009). Rehospitalizations among patients in the medicare fee-for-service program. New England Journal of Medicine, 360, 1418 1428. doi: 10.1056/NEJMsa0803563

Jiang, H., \& Wier, L. (2010). All-cause hospital readmissions among non-elderly Medicaid patients, 2007. Healthcare Cost and Utilization Project, 89, 1-10.

Kaboli, P., Go, J. T., Hockenberry, J., Glasgow, J. M. Johnson, S. R., Rosenthal, G. E. ... Vaughan-Sarrazin, M. (2012). Associations between reduced hospital length of stay and 30-day readmission rate and mortality: 14-year experience in 129 veterans affairs hospitals. Annals of Internal Medicine, 157, 837-845.

Kociol, R. (2011). Hospital readmission after myocardial infarction: A distinctly American problem? Poster presentation presented at the American Heart Association Scientific Sessions, Orlando, FL.

Kociol, R., Liang, L., Hernandez, A., Curtis, L., Heidenreich, P., Yancy, C. . . Peterson, E. D. (2013). Are we targeting the right metric for heart failure? Comparison of hospital 30-day readmission rates and total episode of care inpatient days. American Heart Journal, 165, 987-994. Retrieved from http://dx.doi.org/10.1016/j.ahj.2013.02.006

Kociol, R., Lopes, R., Clare, R., Thomas, L., Mehta, R., \& Kaul, P. (2012). International variation in and factors associated with hospital readmission after myocardial infarction. Journal of the American Medical Association, 307, 66-74. 
Kuklina, E., Shaw, K., \& Hong, Y. (2011). Vital signs: Prevalence, treatment and control of high levels of low-density lipoprotein cholesterol-United States, 1999-2002 and 2005-2008. Morbidity and Mortality Weekly Report, 60, 109-114.

Lavie, C. J., \& Milani, R. V. (2004). Cardiac rehabilitation and depression. American Journal of Cardiology, 93(8), 1080.

Lavie, C. J., Milani, R. V., Cassidy, M. M., \& Gilliland, Y. E. (1999). Benefits of cardiac rehabilitation and exercise training in women with depression. American Journal of Cardiology, 83, 1480-1483.

Lavie, C., Morshedi-Meibodi, A., \& Milani, R. (2008). Impact of Cardiac rehabilitation on coronary risk factors, inflammation, and the metabolic syndrome in obese coronary patients. Journal of Cardiometabolic Syndrome, 3, 136-140.

Lavie, C., Thomas, R., Squires, R., Allison, T., \& Milani, R. (2009). Exercise training and cardiac rehabilitation in primary and secondary prevention of coronary heart disease. Mayo Clinic Proceedings, 84, 373-383.

Lett, H., Blumenthal, J., Babyak, M., Sherwood, A., Strauman, T., Robins, C., \& Newman, M. F. (2004). Depression as a risk factor for coronary artery disease: Evidence, mechanisms, and treatment. Psychosomatic Medicine, 66, 305-315. Lichtman, J., Bigger, T., Blumenthal, J., Frasure-Smith, N., Kaufmann, P., Lesperance, F. ... Froeliher, E. S. (2008). Depression and coronary heart disease: recommendations for screening, referral and treatment: A science advisory from the American Heart Association Prevention Committee of the Council on Cardiovascular Nursing, Council on Clinical Cardiology, Council on 
Epidemiology and Prevention, and Interdisciplinary Council on Quality of Care and Outcomes Research: Endorsed by the American Psychiatric Association. Circulation, 11, 1768-1775. doi: 10.1161/circulationaha.108.190769

Lucini, D., Milani, R., Costantino, G., Lavie, C., Porta, A., \& Pagani, M. (2002). Effects of cardiac rehabilitation and exercise training on autonomic regulation in patients with coronary artery disease. American Heart Journal, 143, 977-983.

Mazzini, M., Stevens, G., Whalen, D., Ozonoff, A., \& Balady, G. (2008). Effect of an American Heart Association Get With The Guidelines Program-Based clinical pathway on referral and enrollment into cardiac rehabilitation after myocardial infarction. American Journal of Cardiology, 101, 1084-1087. doi: 10.1016/j.amjcard.2007.11.063.

McGill, H., McMahan, A., Zieske, A., Sloop, G., Walcott, J., Troxclair, D. . . Strong, J. P. (2000). Associations of coronary heart disease risk factors with the intermediate lesion of atherosclerosis in youth. Arteriosclerosis Thrombosis Vascular Biology, 20, 1998-2004. doi: 10.1161/01.ATV.20.8.1998

Medicare Payment Advisory Commission. (2007). Report to the Congress: Promoting greater efficiency in medicine. Washington, DC: Author.

Milani, R., \& Lavie, C. (2003). Prevalence and profile of metabolic syndrome in patients following acute coronary events and effects of therapeutic lifestyle change with cardiac rehabilitation. American Journal of Cardiology, 92, 50-54.

Milani, R., \& Lavie, C. (2007). Impact of cardiac rehabilitation on depression and its associated mortality. American Journal of Medicine, 120, 799-806. 
Milani, R., Lavie, C., \& Cassidy, M. (1996). Effects of cardiac rehabilitation and exercise training programs on depression in patients after major coronary events. American Heart Journal, 132, 726-732.

Milani, R., Lavie, C., \& Mehra, M. (2004). Reduction in c-reactive protein through cardiac rehabilitation and exercise training. Journal of American College of Cardiology, 43, 1056-1061.

Neubeck, L., Freedman, S., Clark, A., Briffa, T., Bauman, A., \& Redfern, J. (2011). Participating in cardiac rehabilitation: A systematic review and meta-synthesis of qualitative data. European Journal of Cardiovascular Prevention and Rehabilitation, 19, 494-503. doi: 10.1177/1741826711409326

Ogden, C., Carroll, M., Kit, B., \& Flegal, K. (2012). Prevalence of obesity in the United States, 2009-2010. National Center for Health Statistics, 82 (DHHS Publication No. [PHS] 2012-1209).

Ostchega, Y., Yoon, S., Hughes, J., \& Louis, T. (2008). Hypertension awareness, treatment and control-continued disparities in adults: United States, 2005-2006. (National Center for Health Statistics Data Brief No. 3), Hyattsville, MD: National Center for Health Statistics.

Plüss, C., Billing, E., Held, C., Henriksson, P., Kiessling, A., Karlsson, M., \& Wallen, H. N. (2011). Long-term effects of an expanded cardiac rehabilitation programme after myocardial infarction or coronary artery bypass surgery: A five-year followup of a randomized controlled study. Clinical Rehabilitation, 25, 79-87. doi: $10.1177 / 0269215510376006$ 
Ricciardi, M., Selzer, F., Marroquin, O., Holper, E., Venkitachalam, L., Williams, D. . . Laskey, W. K. (2012). Incidence and predictors of 30-day hospital readmission rate following percutaneous coronary intervention (From the National Heart, Lung, and Blood Institute Dynamic Registry). American Journal of Cardiology, 110(10), 1389-1396. doi: 10.1016/j.amjcar.2012.07.002

Rozanski, A., Blumenthal, J., \& Kaplan, J. (1999). Impact of psychological factors on the pathogenesis of cardiovascular disease and implications for therapy. Circulation, 99, 2192-2217.

Rozanski, A., Blumenthal, J., Davidson, K., Saab, P., \& Kubzansky, L. (2005). The epidemiology, pathophysiology, and management of psychosocial risk factors in cardiac practice: The emerging field of behavioral cardiology. Journal of the American College of Cardiology, 45, 637-651.

Rugulies, R. (2002). Depression as a predictor for coronary artery disease: A review and meta-analysis 1. American Journal of Preventative Medicine, 23, 51-61.

Schiller, J., Lucas, J., Ward, B., \& Peregoy, J. (2012). Summary health statistics for U.S. adults: National Health Interview Survey, 2010. Vital Health Statistics 10(252).

Sniderman, A., Pedersen, T., \& Kjekshus, J. (1997). Putting low-density lipoproteins at center stage in atherogenesis. American Journal of Cardiology, 79, 64-67.

Squires, R. W., \& Hamm, L. F. (2006). Exercise and the coronary heart disease connection. In: L. F. Hamm LF, K. Berra K, T. Kavanagh T. (Eds.), AACVPR Cardiac Rehabilitation Resource Manual (pp. 53-62). Champaign, IL: Human Kinetics. 
Squires, R., Gau, G., Miller, T., Allison, T., \& Lavie, C. (1990). Cardiovascular rehabilitation: Status, 1990. Mayo Clinic Proceedings, 65, 731-755. doi: $10.1016 / \mathrm{S} 0025-6196(12) 65134-9$

Stewart, R., Campos, C., Jennings, B., Lollis, S., Levitsky, S., \& Lahey, S. (2000). Predictors of 30-day hospital readmission after coronary artery bypass. Annals of Thoracic Surgery, 70, 169-174.

Suaya, J., Shepard, D., Normand, S., Ades, P., Prottas, J., \& Stason, W. (2007). Use of cardiac rehabilitation by medicare beneficiaries after myocardial infarction or coronary bypass surgery. Circulation, 116, 1653-1662. doi:

\subsection{1/circulationaha.107.701466}

The Patient Protection and Affordable Care Act (PPACA), P.L. 111-148, enacted on March 23, 2010 and the Health Care and Education Reconciliation Act of 2010 (HCERA), P.L. 111-152, enacted on March 30, 2010. See: Section 3025.

The Patient Protection and Affordable Care Act (PPACA), P.L. 111-148, enacted on March 23, 2010 and the Health Care and Education Reconciliation Act of 2010 (HCERA), P.L. 111-152, enacted on March 30, 2010. See: Section 3025.

Thomas, R. (2007). Cardiac rehabilitation/secondary prevention programs. A raft for the rapids: Why have we missed the boat? Circulation, 116, 1644-1646.

Thomas, R., King, M., Lui, K., Oldridge, N., Pina, I. L., Spertus, J. . . Whitman, G. R. (2007). AACVPR/ACC/AHA 2007 performance measures on cardiac rehabilitation for referral to and delivery of cardiac rehabilitation/secondary prevention services endorsed by the American College of Chest Physicians, 
American College of Sports Medicine, American Physical Therapy Association, Canadian Association of Cardiac Rehabilitation, European Association for Cardiovascular Prevention and Rehabilitation, Inter-American Heart Foundation, National Association of Clinical Nurse Specialists, Preventive Cardiovascular Nurses Association, and the Society of Thoracic Surgeons. Journal of the American College of Cardiology, 50, 1400-1433. doi: 10.1016/j.jacc2007.04.033

Thomas, R., Miller, N., Lamendola, C., Berra, K., Hedback, B., Durstine, J. L., \& Haskell, W. L. (1996). National survey on gender differences in cardiac rehabilitation programs: Patient characteristics and enrollment patterns. Journal of Cardiopulmonary Rehabilitation, 16, 402-412.

Thomas, R., Witt, B., Lopez-Jimenez, F., King, M., \& Squires, R. (2005). Quality indicators in cardiovascular care: The case for cardiac rehabilitation. Journal of Cardiopulmonary Rehabilitation, 25, 249-256.

Tiller, S., Leger-Caldwell, L., O’Farrell, P., Pipe, A., \& Mark, A. (2013). Cardiac rehabilitation beginning at the bedside. Journal of Cardiopulmonary Rehabilitation, $x x$, 1-5. doi: 10.1097/HCR/0b013e31827e14da

Valencia, H., Savage, P., \& Ades, P. (2011). Cardiac rehabilitation in underserved populations. Minorities, low socioeconomic, and rural residents. Journal of Cardiopulmonary Rehabilitation and Prevention, 31, 203-210. doi: 10.1097/HCR.0v013e318220a7da

Vogel, R. (1997). Coronary risk factors, endothelial function, and atherosclerosis: S review. Clinical Cardiology, 20, 426-432. 
Wier, L., Barrett, M., Steiner, C., \& Jiang, J. (2011). All-cause readmissions by payer and age, 2008. Healthcare Cost and Utilization Project, 115, 1-9.

Williams, D., Holubkov, R., Yeh, W., Bourassa, M., Al-Bassam, M., Block, P. . . Detre, K. M. (2000). Percutaneous coronary intervention in the current era compared with 1985-1986: The National Heart, Lung, and Blood Institute Registries.

Circulation, 102, 2945-2951. doi: 10.1161/01.CIR.102.24.2945

Williams, M., Ades, P., Hamm, L., Keteyian, S., LaFontaine, T., Roitman, J., \& Squires, R. W. (2006). Clinical evidence for a health benefit from cardiac rehabilitation: An update. American Heart Journal, 152, 835-841. doi:

10.1016/j.ahj.2006.05.015

Witt, B., Thomas, R., \& Roger, V. (2005). Cardiac rehabilitation after myocardial infarction: A review to better understand barriers and potential solutions. Europa Medicophysica, 41, 27-34.

Wulsin, L., \& Singal, B. (2003). do depressive symptoms increase the risk for the onset of coronary disease? A systemic quantitative review. Psychosomatic Medicine, 65, 201-210.

Yabroff, K., Lund, J., Kepka, D., \& Mariotto, A. (2011). Economic burden of cancer in the US: Estimates, projections, and future research. Cancer Epidemiology Biomarkers, 20, 2006-2014. doi: 10.1158/1055-9965.EPI-11-0650

Zhu, J., Nieto, F., Horne, B., Anderson, J., Muhlestein, J., \& Epstein, S. (2001). Prospective study of pathogen burden and risk of myocardial infarction or death. Circulation, 103, 45-50. 